\title{
Packing Lines, Planes, etc.: Packings in Grassmannian Spaces
}

John H. Conway, Ronald H. Hardin and Neil J. A. Sloane

\section{CONTENTS}

1. Introduction

2. Grassmannian Space

3. Packing Lines Through the Origin in Three Dimensions

4. Packing Planes in Four Dimensions

5. Packing n-Planes in Higher Dimensions

6. Packing Lines in Higher Dimensions

Acknowledgements

Electronic Availability

References
We address the question: How should $N n$-dimensional subspaces of $m$-dimensional Euclidean space be arranged so that they are as far apart as possible? The results of extensive computations for modest values of $N, n, m$ are described, as well as a reformulation of the problem that was suggested by these computations. The reformulation gives a way to describe $n$ dimensional subspaces of $m$-space as points on a sphere in dimension $\frac{1}{2}(m-1)(m+2)$, which provides a (usually) lowerdimensional representation than the Plücker embedding, and leads to a proof that many of the new packings are optimal. The results have applications to the graphical display of multidimensional data via Asimov's grand tour method.

\section{INTRODUCTION}

Although there is a considerable literature dealing with Grassmannian spaces, exemplified by [Chow 1949; Leichtweiss 1961; Wong 1967; James and Constantine 1974; Griffiths and Harris 1978, Section 1.5; MacKenzie and Morgan 1995; Zanella 1995], the problem of finding the best packings in such spaces seems to have received little attention.

We have made extensive computations on this problem, and have found a number of putatively optimal packings. These computations have led us to conclude that the best definition of distance on Grassmannian space is the "chordal distance" defined in Section 2.

Sections 3, 4, 5, 6 discuss the problems of packing lines in $\mathbb{R}^{3}$, planes in $\mathbb{R}^{4}, n$-spaces in $\mathbb{R}^{m}$, and lines in $\mathbb{R}^{m}$, respectively. Our search has concentrated on packings of $N \leq 55$ subspaces of $G(m, n)$, for $m \leq 16$ and $n \leq 3$. The results are available electronically: see before the bibliography. 


\section{GRASSMANNIAN SPACE}

Grassmannian space $G(m, n)$ is the set of all $n$ dimensional subspaces of real Euclidean $m$-dimensional space $\mathbb{R}^{m}$. It is a homogeneous space isomorphic to $O(m) /(O(n) \times O(m-n))$, and forms a compact Riemannian manifold of dimension $n(m-n)$.

We start by discussing how to define the distance between two elements of $G(m, n)$. Following [Golub and Van Loan 1989, p. 584], for example, we associate with two $n$-planes $P$ and $Q$ principal angles $\theta_{i} \in[0, \pi / 2]$ and principal vectors $u_{i} \in P$ and $v_{i} \in Q$, for $i=1, \ldots, n$, as follows. Choose $u_{1} \in P$ and $v_{1} \in Q$ having length 1 and such that $u_{1} \cdot v_{1}$ is maximal. Inductively, define $u_{i} \in P$ and $v_{i} \in Q$ having length 1 and such that $u_{i} \cdot v_{i}$ is maximal, subject to the conditions $u_{i} \cdot u_{j}=0$ and $v_{i} \cdot v_{j}=0$ for all $1 \leq j<i$. Then set $\theta_{i}=\arccos u_{i} \cdot v_{i}$.

Wong [1967] shows that the geodesic distance on $G(m, n)$ between $P$ and $Q$ is

$$
d_{g}(P, Q)=\sqrt{\theta_{1}^{2}+\cdots+\theta_{n}^{2}}
$$

This distance function has the drawback of not being everywhere differentiable. Consider the case $n=1$, for example, and hold one line $P$ fixed while rotating another line $Q$ (both passing through the origin). As the angle $\varphi$ between $P$ and $Q$ increases from 0 to $\pi$, the principal angle $\theta_{1}$ increases from 0 to $\pi / 2$ and then falls to 0 , and is nondifferentiable at $\pi / 2$, as illustrated in Figure 1.

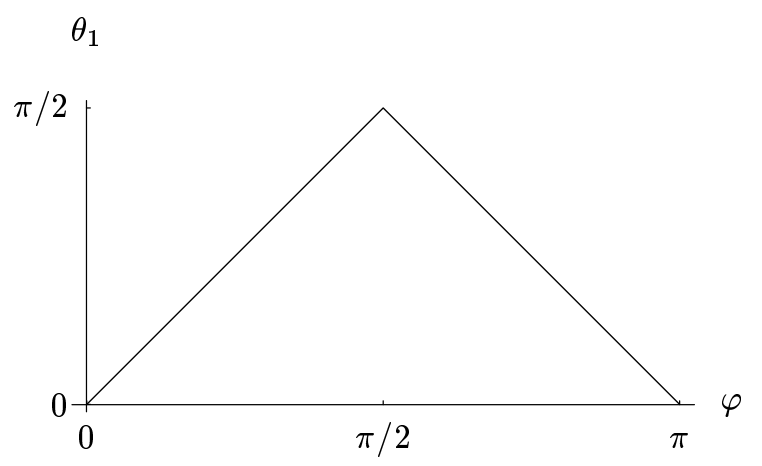

FIGURE 1. Principal angle $\theta_{1}$ between two lines as the angle between them increases from 0 to $\pi$.
Although one might expect this nondifferentiability to be a mere technicality, it causes many difficulties in practice. Our optimizer software does not handle it well, especially in higher dimensions in cases when many distances fall in the neighborhood of singular points of $d_{g}$.

An alternative measure of distance, which we call the chordal distance for reasons to be made clear later, is given by

$$
d_{c}(P, Q)=\sqrt{\sin ^{2} \theta_{1}+\cdots+\sin ^{2} \theta_{n}} .
$$

This approximates the geodesic distance when the planes are close, has the property that its square is differentiable everywhere, and, as we shall attempt to demonstrate, has a number of other desirable features.

A third definition has been used [Asimov 1985; Golub and Van Loan 1989, p. 584], namely

$$
d_{m}(P, Q)=\theta_{n}=\max _{i=1, \ldots, n} \theta_{i}
$$

It shares the vices of the geodesic distance.

Of course, for $n=1$ all three definitions are equivalent, in the sense that they lead to the same optimal packings.

We can now state the packing problem: given $N, n, m$, find a set of $n$-planes $P_{1}, \ldots, P_{N} \in G(m, n)$ such that $\min _{i \neq j} d\left(P_{i}, P_{j}\right)$ is as large as possible, where $d$ is either geodesic or chordal distance. That there is such a set follows from the compactness of $G(m, n)$. Since $G(m, n)$ and $G(m, m-n)$ can be identified by the correspondence induced by orthogonal complementation, we will usually assume that $n \leq m / 2$.

A generator matrix for an $n$-plane $P \in G(m, n)$ is an $n \times m$ matrix whose rows span $P$. The orthogonal group $O(m)$ acts on $G(m, n)$ by right multiplication of generator matrices. The automorphism group of a subset $\left\{P_{1}, \ldots, P_{N}\right\} \subset G(m, n)$ is the subset of $O(m)$ which fixes or permutes these planes.

Applying a suitable element of $O(m)$ and choosing appropriate basis vectors for the planes, we can 
reduce the generator matrices of any given pair of $n$-planes $P, Q$ with $n \leq m / 2$ to the forms

$$
\left[\begin{array}{cccccccc}
1 & 0 & \cdots & 0 & 0 & 0 & \cdots & 0 \\
0 & 1 & \cdots & 0 & 0 & 0 & \cdots & 0 \\
\vdots & \vdots & \ddots & \vdots & \vdots & \vdots & \ddots & \vdots \\
0 & 0 & \cdots & 1 & 0 & 0 & \cdots & 0
\end{array}\right]
$$

and

$\left[\begin{array}{ccccccccccc}\cos \theta_{1} & 0 & \cdots & 0 & \sin \theta_{1} & 0 & \cdots & 0 & 0 & \cdots & 0 \\ 0 & \cos \theta_{2} & \cdots & 0 & 0 & \sin \theta_{2} & \cdots & 0 & 0 & \cdots & 0 \\ \vdots & \vdots & \ddots & \vdots & \vdots & \vdots & \ddots & \vdots & \vdots & \ddots & \vdots \\ 0 & 0 & \cdots & \cos \theta_{n} & 0 & 0 & \cdots & \sin \theta_{n} & 0 & \cdots & 0\end{array}\right]$

respectively, where $\theta_{1}, \ldots, \theta_{n}$ are the principal angles between $P$ and $Q$ [Wong 1967, Theorem 2].

\section{PACKING LINES THROUGH THE ORIGIN IN THREE DIMENSIONS}

Our initial work on this problem was prompted by a question raised by Julian Rosenman [1992], an oncologist at the University of North Carolina School of Medicine and Computer Science, in connection with the treatment of tumors using high energy laser beams. Rosenman asked for the best way to separate $N$ lines through a given point in $\mathbb{R}^{3}$, or in other words for the best packings in $G(3,1)$.

Together with W. D. Smith, we had been carrying out an extensive search for the best packings of a given number of points on $S^{2}$, that is, spherical codes [Hardin et al. 1993-95; a], and we therefore modified our programs to search instead for packings of lines; packing $N$ points in $G(m, 1)$ is the same as packing $2 N$ points in $S^{m-1}$ with the condition that all points form antipodal pairs. We omit the details of this search, since we later redid the calculations using the more general methods described in Section 4.

Table 1 lists, for each value of $N$ from 2 to 55 , the minimum distance $\theta_{1}$ for the best antipodal packing of $2 N$ points on $S^{2}$ that we have found. For comparison, the table also shows the minimal angle for the best known packing of $2 N$ points without the antipodal condition. We see that requiring a packing on $S^{2}$ to be antipodal is a definite handicap: only in the cases $N=3$ and $N=6$ do the antipodal and unrestricted packings coincide.

The table also gives the automorphism group of the antipodal packing, that is, the subgroup of $O(3)$ that leaves the set of points invariant. The groups are given in orbifold notation [Conway and Sloane a] and as the double cover of a rotation group. The symbol $\pm \mathcal{G}$ indicates that the group consists of the matrices $\pm M$ for $M \in \mathcal{G}$, where $\mathcal{G}$ is a cyclic $(\mathcal{C})$, dihedral $(\mathcal{D})$, tetrahedral $(\mathcal{T})$, octahedral $(\mathcal{O})$ or icosahedral $(\mathcal{J})$ group. In each case the subscript gives the order of the rotation group, so the order of the automorphism group is twice this subscript.

We turn to some noteworthy features of certain entries of Table 1.

$N=5$

The five lines are five of the six diameters of a regular icosahedron.

$N=8$

The putative solution forms an unpleasant-looking configuration of 16 antipodal points on $S^{2}$, with no further symmetry. The convex hull is shown on the left in Figure 2. In contrast, the putatively best packing of 16 points, shown on the right in the same figure, has a group of order 16 .
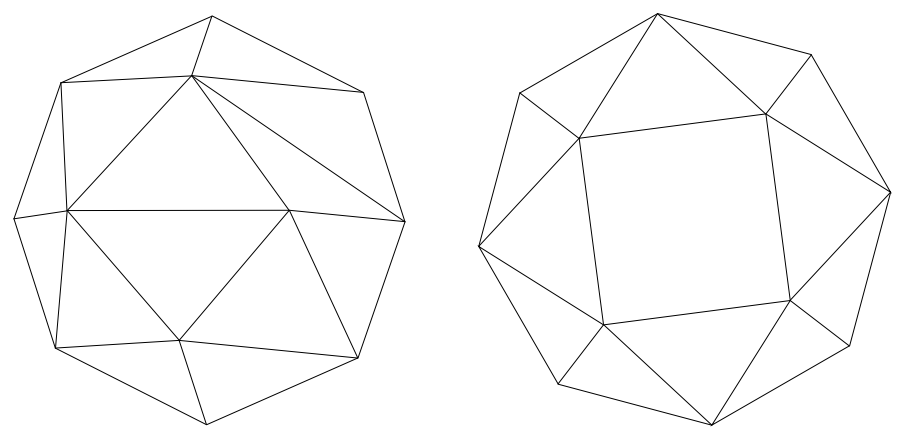

FIGURE 2. $N=8$ : Best antipodal packing known (left) and best unrestricted packing known (right) of 16 points on the sphere. 


\begin{tabular}{|c|c|c|c|c|c|c|c|c|}
\hline$N$ & $\min \theta_{1}$ & $\min \theta_{1}^{\prime}$ & group & polyhedron & $N$ & $\min \theta_{1}$ & $\min \theta_{1}^{\prime}$ & group \\
\hline 2 & 90.0000 & 109.4712 & $* 224= \pm \mathcal{D}_{8}$ & square & 29 & 27.5244 & 27.5564 & $2 * 7= \pm \mathcal{D}_{14}$ \\
\hline 3 & 90.0000 & 90.0000 & $* 432= \pm \mathcal{O}_{24}$ & octahedron & 30 & 26.9983 & 27.1928 & $2 * 5= \pm \mathcal{D}_{10}$ \\
\hline 4 & 70.5288 & 74.8585 & $* 432= \pm \mathcal{O}_{24}$ & cube & 31 & 26.4987 & 26.6840 & $5 \times= \pm \mathcal{C}_{5}$ \\
\hline $5^{*}$ & 63.4349 & 66.1468 & $2 * 5= \pm \mathcal{D}_{10}$ & pentagonal antiprism & 32 & 25.9497 & 26.2350 & $2 *= \pm \mathcal{C}_{2}$ \\
\hline 6 & 63.4349 & 63.4349 & $* 532= \pm \mathcal{J}_{60}$ & icosahedron & 33 & 25.5748 & 25.9474 & $3 * 2= \pm \mathcal{T}_{12}$ \\
\hline 7 & 54.7356 & 55.6706 & $* 432= \pm \mathcal{O}_{24}$ & rhombic dodecahedron & 34 & 25.2567 & 25.4638 & $3 \times= \pm \mathcal{C}_{3}$ \\
\hline $8^{*}$ & 49.6399 & 52.2444 & $\times= \pm \mathcal{C}_{1}$ & & 35 & 24.8702 & 25.1709 & $\times= \pm \mathcal{C}_{1}$ \\
\hline $9 *$ & 47.9821 & 49.5567 & $3 \times= \pm \mathcal{C}_{3}$ & & 36 & 24.5758 & 24.9265 & $3 \times= \pm \mathcal{C}_{3}$ \\
\hline $9^{*}$ & 47.9821 & 49.5567 & $2 *= \pm \mathcal{C}_{2}$ & & 37 & 24.2859 & 24.4209 & $\times= \pm \mathfrak{C}_{1}$ \\
\hline $10^{*}$ & 46.6746 & 47.4310 & $* 226= \pm \mathcal{D}_{12}$ & hexakis bi-antiprism & 38 & 24.0886 & 24.1282 & $x= \pm \mathcal{C}_{1}$ \\
\hline 11 & 44.4031 & 44.7402 & $2 * 5= \pm \mathcal{D}_{10}$ & & 39 & 23.8433 & 23.9310 & $3 \times= \pm \mathcal{C}_{3}$ \\
\hline 12 & 41.8820 & 43.6908 & $* 432= \pm \mathcal{O}_{24}$ & rhombicuboctahedron & 40 & 23.3293 & 23.5531 & $\times= \pm \mathcal{C}_{1}$ \\
\hline 13 & 39.8131 & 41.0377 & $2 *= \pm \mathrm{C}_{2}$ & & 41 & 22.9915 & 23.1946 & $\times= \pm \mathrm{C}_{1}$ \\
\hline 14 & 38.6824 & 39.3551 & $\times= \pm \mathcal{C}_{1}$ & & 42 & 22.7075 & 23.0517 & $3 \times= \pm \mathcal{C}_{3}$ \\
\hline $15^{*}$ & 38.1349 & 38.5971 & $2 * 5= \pm \mathcal{D}_{10}$ & & 43 & 22.5383 & 22.6744 & $2 * 3= \pm \mathcal{D}_{6}$ \\
\hline 16 & 37.3774 & 37.4752 & $* 532= \pm \mathcal{J}_{60}$ & pentakis dodecahedron & 44 & 22.2012 & 22.4679 & $\times= \pm \mathcal{C}_{1}$ \\
\hline 17 & 35.2353 & 35.8078 & $\times= \pm \mathcal{C}_{1}$ & & 45 & 22.0481 & 22.1540 & $2 *= \pm \mathcal{C}_{2}$ \\
\hline 18 & 34.4088 & 35.1897 & $3 \times= \pm \mathcal{C}_{3}$ & & 46 & 21.8426 & 22.0276 & $\times= \pm \mathrm{C}_{1}$ \\
\hline 19 & 33.2115 & 34.2507 & $\times= \pm \mathcal{C}_{1}$ & & 47 & 21.6609 & 21.7221 & $\times= \pm \mathcal{C}_{1}$ \\
\hline 20 & 32.7071 & 33.1584 & $* 222= \pm \mathcal{D}_{4}$ & & 48 & 21.4663 & 21.5206 & $3 \times= \pm \mathcal{C}_{3}$ \\
\hline 21 & 32.2161 & 32.5064 & $5 \times= \pm \mathcal{C}_{5}$ & & 49 & 21.1610 & 21.3711 & $\times= \pm \mathcal{C}_{1}$ \\
\hline 22 & 31.8963 & 31.9834 & $2 * 3= \pm \mathcal{D}_{6}$ & & 50 & 20.8922 & 21.0312 & $\times= \pm \mathcal{C}_{1}$ \\
\hline 23 & 30.5062 & 30.9592 & $\times= \pm \mathfrak{C}_{1}$ & & 51 & 20.6903 & 20.8556 & $\times= \pm \mathfrak{C}_{1}$ \\
\hline 24 & 30.1628 & 30.7628 & $3 * 2= \pm \mathcal{T}_{12}$ & & 52 & 20.4914 & 20.6566 & $\times= \pm \mathfrak{C}_{1}$ \\
\hline 25 & 29.2486 & 29.7530 & $3 \times= \pm \mathcal{C}_{3}$ & & 53 & 20.2685 & 20.4394 & $\times= \pm \mathrm{C}_{1}$ \\
\hline 26 & 28.7126 & 29.1948 & $2 *= \pm \mathcal{C}_{2}$ & & 54 & 20.1555 & 20.3044 & $3 \times= \pm \mathcal{C}_{3}$ \\
\hline 27 & 28.2495 & 28.7169 & $\times= \pm \mathrm{C}_{1}$ & & $55^{*}$ & 20.1034 & 20.1110 & $* 532= \pm \mathcal{J}_{60}$ \\
\hline 28 & 27.8473 & 28.1480 & $\times= \pm \mathrm{C}_{1}$ & & & & & \\
\hline
\end{tabular}

TABLE 1. Summary of results about packings in $G(3,1)$. For each $N$ we give, rounded to four decimal places, the minimal angle $\theta_{1}$ of the best packing we could find of $N$ points in $G(3,1)$ (that is, the best packing of $N$ lines through the origin in $\mathbb{R}^{3}$, or the best antipodal packing of $2 N$ points on $S^{2}$ ), and the minimal angle $\theta_{1}^{\prime}$ of the best known unconstrained packing of $2 N$ points on $S^{2}$, taken from [Hardin et al. 1993-95]. The fourth column shows the automorphism group for the best antipodal packing, both in orbifold notation and as a double cover of a rotation group (see text for notation). When the packing corresponds to the diameters of a known polyhedron, this is also indicated. An asterisk next to the value of $N$ refers to notes and figures starting on page 141.

The entries for $N \leq 6$ were shown to be optimal by Fejes Tóth [1965]; see also [Rosenfeld 1994]. The case $N=7$ will be proved in Section 5 . The solutions for $N \geq 8$ are the best found with over 15000 random starts with our optimizer. There is no guarantee that they are optimal, but experience with similar problems suggests that they will be hard to beat and in any case will be not far from optimal.

For $N=1,2,3,6$ the solutions are known to be unique, for $N=4$ there are precisely two solutions [Fejes Tóth 1965; Rosenfeld 1993; Rosenfeld 1994], and for $N=5,7,8$ the solutions appear to be unique. For larger values of $N$, however, the solutions are often not unique. For $N=9$ lines there are two different solutions, and in the range $N \leq 30$ the solutions for 10,22, 25,27, 29 lines (and possibly others) contain lines that "rattle", that is, lines that can be moved freely over a small range of angles without affecting the minimal angle.

Remark: Entries in all tables are approximated by rounding, rather than truncation. 
$\mathrm{N}=9$

There appear to be two inequivalent solutions. The nicer one has an order- 6 symmetry group, $3 \times$, or $\left[2^{+}, 6^{+}\right]$in Coxeter's notation [Coxeter and Moser 1980]; see Figure 3, top. The points lie in six parallel layers of equilateral triangles.

The second solution has group $2 *$, of order 4 ; see Figure 3, bottom.
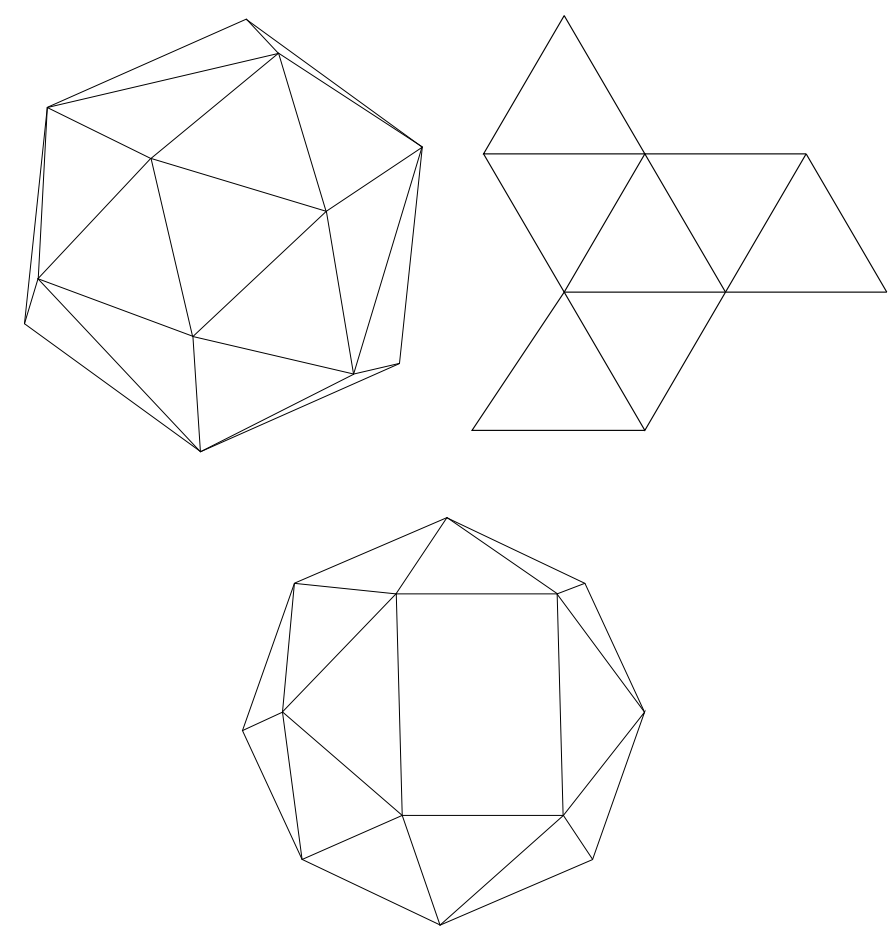

FIGURE 3. $N=9$ : Antipodal packings of 18 points with group of order 6 (top) and order 4 (bottom). In the order- 6 arrangement, half the points can be located by wrapping a pinwheel of seven equilateral triangles (top right) onto the sphere, so that the edges of the spherical triangles have length $47.98213264^{\circ}$.

$N=10$

This is a hexakis bi-antiprism, since it consists of a bi-(hexagonal antiprism) together with an axial line that can rattle, giving infinitely many solutions. The axis appears horizontally in Figure 4, left.
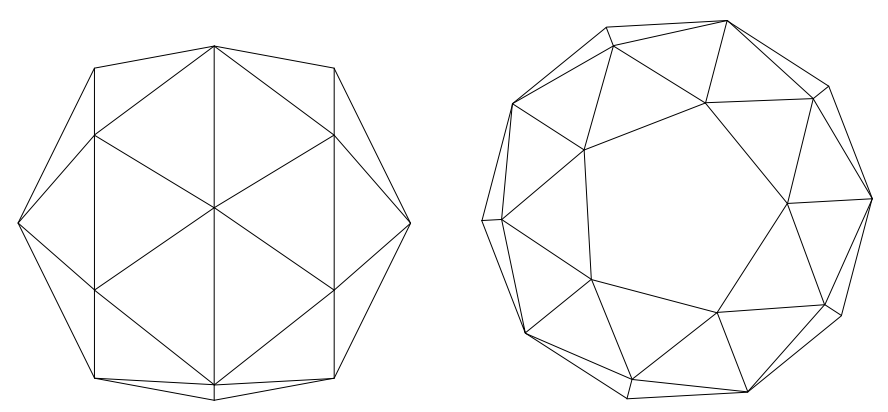

FIGURE 4. $N=10,15$ : Best antipodal packing found for 20 points (left) and for 30 points (right).

$\mathrm{N}=15$

Combinatorially, this arrangement is obtained from a pentakis dodecahedron (the solution for $N=16$ ) by omitting two opposite vertices, as shown in Figure 4, right. However, the angular separation is slightly greater than for $N=16$.

$N=55$

The 110 antipodal points can be taken to be the union of the vertex sets of a dodecahedron (20), an icosidodecahedron (30) and a truncated icosahedron (60), as shown in Figure 5. The 15 lines through the icosidodecahedral points rattle. This arrangement can be obtained from a geodesic dome or 12-fold reticulated icosahedron [Coxeter 1974] by omitting its twelve pentagonal vertices. Inci-

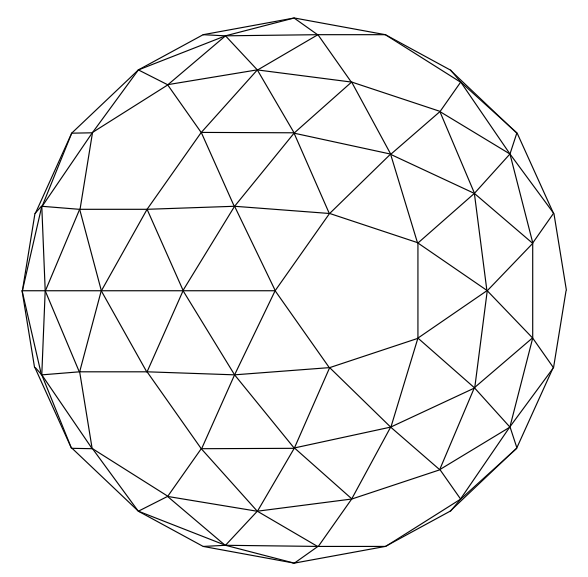

FIGURE 5. Best antipodal packing found of 110 points. 
dentally, our best solution for 61 lines has a symmetry group of order 6 , and is not found by placing points at the centers of the pentagonal faces of Figure 5.

Packings of lines in higher dimensional spaces will be discussed in Section 6 .

\section{PACKING PLANES IN FOUR DIMENSIONS}

Asimov [1985] has proposed a technique called the grand tour for displaying multi-dimensional data on a two-dimensional computer screen. His idea was to choose a finite sequence, or tour, of twodimensional planes that are in some sense representative of all planes, and to project the data onto each plane in turn, in the hope that the viewer will be able to notice any pattern or structure that is present. This technique has been implemented in the XGobi program [Buja and Asimov 1986; Swayne et al. 1991].

In 1993 Dianne Cook asked us if we could modify our algorithm for finding spherical codes in order to search for packings in $G(m, 2)$ for $m \geq 4$. Furthermore, for the grand tour application, there should be a Hamiltonian circuit through the planes, in which edges indicate neighboring planes. We accomplished this in the following way.

Following the methods we had used to find packings in $S^{d}$ and experimental designs in different spaces [Hardin and Sloane 1992, Section 4.11; 1993; Hardin et al. a], corresponding to a set of planes $S=\left\{P_{1}, \ldots, P_{N}\right\} \subseteq G(m, n)$ we define a potential

$$
\Phi_{c}(S)=\sum_{i<j} \frac{1}{d_{c}\left(P_{i}, P_{j}\right)-A},
$$

where $A$ is a suitably chosen constant. There is a similar definition for $\Phi_{g}$ involving $d_{g}$. Initially $A$ is set to 0 and $S$ is a randomly chosen set of planes. We invoke the Hooke-Jeeves pattern search optimization algorithm to modify $S$, attempting to minimize $\Phi_{c}(S)$. After a fixed time (we used 100 steps of the optimizer), $A$ is advanced halfway to the current minimal distance between the $P_{i}$, and the process is repeated, terminating when no further improvement is obtained to the accuracy of the machine. The whole process is repeated with several thousand random starts - and also with initial configurations taken from other sources, such as subsets or supersets of other arrangements - and the best final configuration is recorded.

The partial derivatives of $\Phi_{c}(S)$ were found analytically, but for $\Phi_{g}(S)$ we calculated them by numerical differentiation.

We began by computing a table of packings of $N$ planes in $G(4,2)$, for $N \leq 50$, with respect to both $d_{c}$ and $d_{g}$. The results for packings of $N \leq 24$ planes are summarized in Table 2 .

Our understanding of these results was hindered by the fact that planes are, well, plain, with no

\begin{tabular}{|r|cc|cc|}
\hline & \multicolumn{2}{|c|}{$d_{c}$-optimal } & \multicolumn{2}{c|}{$d_{g}$-optimal } \\
& $\min d_{c}^{2}$ & $\min d_{g}^{2}$ & $\min d_{c}^{2}$ & $\min d_{g}^{2}$ \\
\hline 2 & 2.0000 & 4.9348 & 2.0000 & 4.9348 \\
3 & 1.5000 & 2.1932 & 1.2500 & 2.7416 \\
4 & 1.3333 & 1.8253 & 1.2000 & 2.6824 \\
5 & 1.2500 & 1.9739 & 1.2000 & 2.6824 \\
6 & 1.2000 & 2.6824 & 1.2000 & 2.6824 \\
7 & 1.1667 & 1.6440 & 0.9875 & 2.1281 \\
8 & 1.1429 & 1.5818 & 0.9700 & 1.9235 \\
9 & 1.1231 & 1.5175 & 0.9501 & 1.8087 \\
10 & 1.1111 & 1.5725 & 0.9764 & 1.7886 \\
11 & 1.0000 & 1.2715 & 0.9247 & 1.6711 \\
12 & 1.0000 & 1.3413 & 0.9204 & 1.6416 \\
13 & 1.0000 & 1.2348 & 0.9133 & 1.5600 \\
14 & 1.0000 & 1.2337 & 0.8933 & 1.5327 \\
15 & 1.0000 & 1.2337 & 0.8923 & 1.5284 \\
16 & 1.0000 & 1.2337 & 0.8904 & 1.5210 \\
17 & 1.0000 & 1.2337 & 0.8549 & 1.3925 \\
18 & 1.0000 & 1.2337 & 0.8504 & 1.3768 \\
19 & 0.9091 & 1.1666 & 0.8412 & 1.3477 \\
20 & 0.9091 & 1.1666 & 0.8351 & 1.3284 \\
21 & 0.8684 & 1.0352 & 0.8225 & 1.2834 \\
22 & 0.8629 & 1.0592 & 0.8046 & 1.2385 \\
23 & 0.8451 & 1.0081 & 0.7910 & 1.2012 \\
24 & 0.8372 & 0.9901 & 0.7812 & 1.1707 \\
\hline
\end{tabular}

TABLE 2. Putatively best packings of $N$ planes in $G(4,2)$ with respect to the chordal $\left(d_{c}\right)$ and geodesic $\left(d_{g}\right)$ metrics. 
distinguishing features, and when produced by the computer appear as random generator matrices referred to a random coordinate frame. However, we found that the set of principal vectors in the planes could often be used to find a coordinate system that would reveal some of the structure of the planes.

For two planes the best packing is the same for both metrics, and consists of two mutually orthogonal planes with principal angles $\theta_{1}=\theta_{2}=\pi / 2$, so $d_{c}^{2}=2$ and $d_{g}^{2}=\pi^{2} / 2$.

For three planes the two answers are different. The best packing for chordal distance has generator matrices

$\begin{array}{cccc}1 & 0 & 1 & 0 \\ 0 & 1 & 0 & 1 \\ 1 & 0 & -\frac{1}{2} & \frac{\sqrt{3}}{2} \\ 0 & 1 & -\frac{\sqrt{3}}{2} & -\frac{1}{2} \\ 1 & 0 & -\frac{1}{2} & -\frac{\sqrt{3}}{2} \\ 0 & 1 & \frac{\sqrt{3}}{2} & -\frac{1}{2}\end{array}$

so the principal angles are $\theta_{1}=\theta_{2}=\pi / 3$ between each pair of planes, and $d_{c}^{2}=\frac{3}{2}, d_{g}^{2}=\frac{2}{9} \pi^{2}$. The best packing for geodesic distance has generator matrices

$\begin{array}{cccc}1 & 0 & 0 & 0 \\ 0 & -\frac{1}{\sqrt{2}} & 0 & \frac{1}{\sqrt{2}} \\ 0 & 1 & 0 & 0 \\ 0 & 0 & -\frac{1}{\sqrt{2}} & \frac{1}{\sqrt{2}} \\ 0 & 0 & 1 & 0 \\ -\frac{1}{\sqrt{2}} & 0 & 0 & \frac{1}{\sqrt{2}}\end{array}$

so the principal angles are $\theta_{1}=\pi / 6, \theta_{2}=\pi / 2$, and $d_{c}^{2}=\frac{5}{4}, d_{g}^{2}=\frac{5}{18} \pi^{2}$.

Postponing discussion of $N=4$ and 5 for the moment, we consider the case of six planes, where we discovered that the answer for both metrics formed a regular simplex (although not an isoclinic configuration; compare [Wong 1961], which considers a related problem). The principal angles between every pair of planes were $\theta_{1}=\arcsin 1 / \sqrt{5}$ and $\theta_{2}=\pi / 2$. This set of planes is conveniently described using simplicial coordinates. Let $A, B$, $C, D, E$ be the vectors from the center of a regular simplex in $\mathbb{R}^{4}$ to its vertices, with $A+B+$ $C+D+E=0$, and write $[a, b, c, d, e]$ for $a A+$ $b B+c C+d D+e E$. Then one of the six planes is spanned by $[1, \tau, 1,0,0]$ and its cyclic shifts, where $\tau=\frac{1}{2}(1+\sqrt{5})$, and the other five planes are obtained from it by taking even permutations of these coordinates. (We later found an equally good packing with respect to chordal distance that was not a simplex with respect to geodesic distance. This is described below.)

The six planes intersect the surface of the unit ball of $\mathbb{R}^{4}$ in a remarkable link, the hexalink. It can be shown that, apart from the $n$-component unlinks and Hopf links, which exist for all $n$, the hexalink is the only link of circular rings in which there are orientation-preserving symmetries taking any two links to any other two. (Details will be given elsewhere.)

The discovery of this 6-vertex simplex was initially somewhat of a surprise, since $G(4,2)$ is only a 4-dimensional manifold. It did suggest that the answer should somehow be related to the six equiangular diameters of the icosahedron, and led to the following reformulation of the problem.

We remind the reader that any element $\alpha$ of $S O(4)$ may be represented as $\alpha: x \mapsto \bar{l} x r$, where $x=x_{0}+x_{1} i+x_{2} j+x_{3} k$ represents a point on $S^{3}$ and $l, r$ are unit quaternions [Du Val 1964]. The pair $-l,-r$ represent the same $\alpha$. The correspondence between $\alpha$ and $\pm(l, r)$ is one-to-one.

Given a plane $P \in G(4,2)$, let $\alpha$ be the element of $S O(4)$ that fixes $P$ and negates the points of the orthogonal plane $P^{\perp}$. Then $\alpha^{2}=1$, and for this $\alpha$, it is easy to see that $l=l_{1} i+l_{2} j+l_{3} k$ and $r=r_{1} i+$ $r_{2} j+r_{3} k$ are purely imaginary unit quaternions. This establishes the following result, which can be found in [Leichtweiss 1961], for example.

Theorem 4.1. A plane $P \in G(4,2)$ is represented by a pair $(l, r) \in S^{2} \times S^{2}$, with $(-l,-r)$ representing the same $P$. 
There are simple formulas relating $P$ and $(l, r)$, pointed out to us by Simon Kochen. Given $(l, r)$, if $l \neq-r$ then $P$ is spanned by the vectors corresponding to the quaternions $u=1-l r$ and $v=$ $l+r$. The special case when $l=r$ is even simpler: take $u=1, v=l$. If $l=-r$, take $u$ and $v$ to be purely imaginary unit quaternions such that $l, u, v$ correspond to a coordinate frame. Conversely, if $P$ is spanned by two orthogonal unit vectors represented by quaternions $u, v$, then $\pm(l, r)=$ $\pm(u \bar{v}-v \bar{u}, \bar{v} u-\bar{u} v)$.

Given two planes $P, Q \in G(4,2)$, represented by $\pm(l, r)$ and $\pm\left(l^{\prime}, r^{\prime}\right)$, the principal angles $\theta_{1}, \theta_{2}$ between them may be found as follows. Let $\varphi$ be the angle between $l$ and $l^{\prime}$, and let $\psi$ be the angle between $r$ and $r^{\prime}$, with $0 \leq \varphi \leq \pi$ and $0 \leq \psi \leq \pi$. If $\varphi+\psi>\pi$, replace $\varphi$ by $\pi-\varphi$ and $\psi$ by $\pi-\psi$, so that $0 \leq \varphi+\psi \leq \pi$, with $\varphi \leq \psi$ (say). Then

$$
\begin{array}{ll}
\theta_{1}=\frac{1}{2}(\psi-\varphi), & d_{g}^{2}(P, Q)=\frac{1}{2}\left(\psi^{2}+\varphi^{2}\right), \\
\theta_{2}=\frac{1}{2}(\psi+\varphi), & d_{c}^{2}(P, Q)=1-\cos \psi \cos \varphi
\end{array}
$$

We omit the elementary proof.

A set $S=\left\{P_{1}, \ldots, P_{N}\right\} \subseteq G(4,2)$ is thus represented by a binocular code consisting of a set of pairs $\pm\left(l_{i}, r_{i}\right) \in S^{2} \times S^{2}$. We call the list of $2 N$ points $\pm l_{i}$ (which need not be distinct) the left code corresponding to $S$, and the points $\pm r_{i}$ the right code. Conversely, given two multisets $L \subseteq S^{2}$ and $R \subseteq S^{2}$, each of size $2 N$ and closed under negation, and a bijection or matching $f$ between them that satisfies $f(-l)=-f(l)$ for $l \in L$, we obtain a set of $N$ planes in $G(4,2)$.

The binocular codes for the $d_{c^{-}}$optimal packings of 2 to 5 planes are shown in Figure 6 ; they can all be made to lie in the equatorial planes. Except for $N=3$, the left and right codes are identical. For $N \leq 4$ there are repeated points.

For $N=6$ the left and right codes consist of the twelve vertices of an icosahedron (Figure 7). Setting $\lambda=1 / \sqrt{\tau+2}$ we can take the points to be

$$
\lambda(0, \pm 1, \pm \tau), \quad \lambda( \pm \tau, 0, \pm 1), \quad \lambda( \pm 1, \pm \tau, 0) .
$$
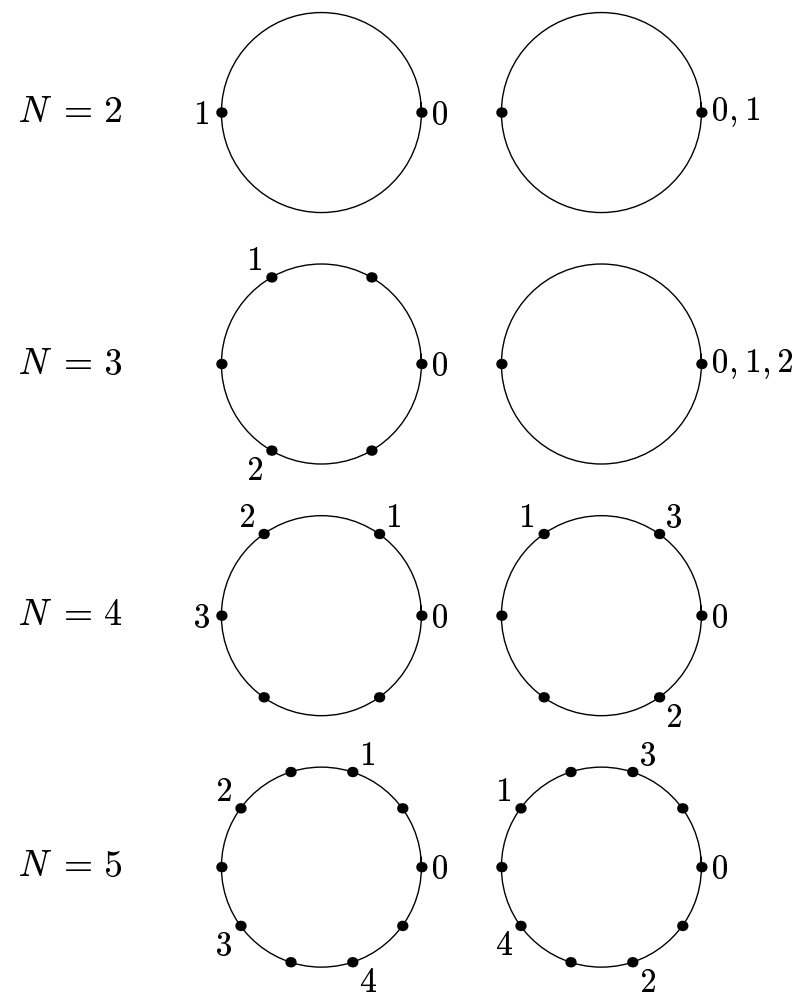

FIGURE 6. Equatorial planes showing the binocular codes describing best packings of 2 to 5 planes for chordal distance. Matching points are labeled with the same symbol. The points for $N=3$ and $N=5$ are vertices of the obvious regular polygons, but for $N=4$ the coordinates are $(1,0)$ and $\pm \frac{1}{\sqrt{3}}(1, \pm \sqrt{2})$.
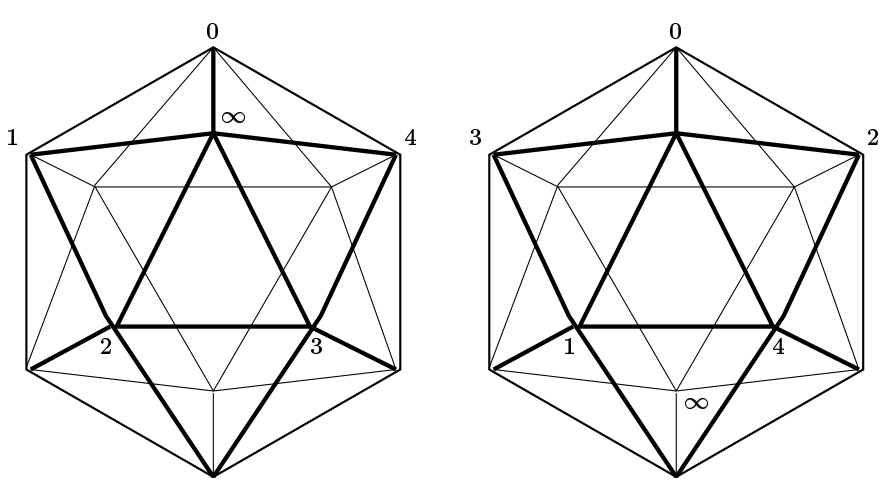

FIGURE 7. Best packing of six planes in $G(4,2)$ with respect to both metrics. Left and right codes comprise vertices of icosahedron. Adjacent vertices in one code are matched with nonadjacent vertices in the other code. 
The matching is obtained by mapping each point to its algebraic conjugate (that is, replacing $\sqrt{5}$ by $-\sqrt{5})$, and rescaling so the points again lie on a unit sphere. As already mentioned, the principal angles between each pair of these planes are $\theta_{1}=$ $\arcsin 1 / \sqrt{5}$ and $\theta_{2}=\pi / 2$, so $d_{c}^{2}=\frac{6}{5}$ and $d_{g}^{2}=$ 2.6824 .

There is a second set of six planes with $d_{c}^{2}=\frac{6}{5}$, but with $d_{g}^{2}$ only equal to 2.0030 . Here the left and right codes form the vertices of what we shall call the antiicosahedron, consisting of the points

$$
\lambda(0, \pm 1, \pm \tau), \quad \lambda( \pm \tau, 0, \pm 1), \quad \lambda( \pm \tau, \pm 1,0)
$$

(compare (4.2)), and shown in Figure 8. Topologically this is a "parallel bi-slit cuboctahedron", obtained by dividing two opposite square faces of a cuboctahedron into two triangles by parallel lines. The matching is this:

$$
\begin{aligned}
& \lambda(0, \pm 1, \tau) \leftrightarrow \lambda(0, \pm \tau, 1), \\
& \lambda(\tau, 0, \pm 1) \leftrightarrow \lambda(1,0, \pm \tau), \\
& \lambda( \pm \tau, 1,0) \leftrightarrow \lambda( \pm 1, \tau, 0) .
\end{aligned}
$$
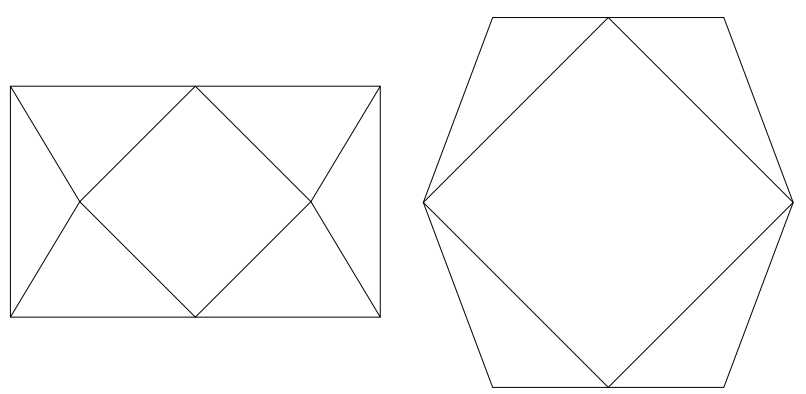

FIGURE 8. Top and side views of antiicosahedron.

Also worth mentioning are four larger putatively $d_{c^{-}}$optimal packings found by the computer, and having 10, 18, 48 and 50 planes.

$N=10$

For the 10-plane packing the left and right codes consist of the vertices of a decagonal prism with coordinates

$$
\pm\left(\sqrt{\frac{2}{3}} \cos r \theta, \sqrt{\frac{2}{3}} \sin r \theta, \sqrt{\frac{1}{3}}\right),
$$

where $\theta=\pi / 5$ and $r=0, \ldots, 9$. A typical point $(\sqrt{2 / 3} \cos r \theta, \sqrt{2 / 3} \sin r \theta, \sqrt{1 / 3})$ in the left code is matched with

$$
(-1)^{r}\left(\sqrt{\frac{2}{3}} \cos 3 r \theta, \sqrt{\frac{2}{3}} \sin 3 r \theta, \sqrt{\frac{1}{3}}\right)
$$

in the right code. Then $d_{c}^{2}=\frac{10}{9}$ and $d_{g}^{2}=1.5725$. The most interesting property of this configuration is that, although three sets of canonical angles occur, the chordal distance between every pair of planes is the same - this is a regular simplex!

We find it surprising that this is superior to the packing of ten planes obtained by matching the vertices of a dodecahedron to their algebraic conjugates, as shown in Figure 9.

$N=18$

Let $\mathcal{O}$ denote the set of vertices of a regular octahedron. The binocular code for the 18-plane packing is $\mathcal{O} \times \mathcal{O}:=\{(l, r): l, r \in \mathcal{O}\}$. Projectively, the 18 two-spaces are the edges of a desmic triple of tetrahedra [Stephanos 1879; Hudson 1905; Coxeter 1950]. (Two tetrahedra are called desmic if they are in perspective from four distinct points; these points then form a third tetrahedron desmic with either of the first two. The 18 edges of such a desmic triple are also the edges of a unique other desmic triple.) We were pleased to see this configuration appear, since it was already familiar in the context of quantum logic [Conway and Kochen]. This set of planes can be constructed directly by taking the planes spanned by all pairs of mutually perpendicular minimal vectors of the $D_{4}$ lattice (compare [Conway and Sloane 1996]). In this form the planes have generator matrices such as

$$
\begin{aligned}
& {\left[\begin{array}{cccc}
+ & + & 0 & 0 \\
0 & 0 & + & -
\end{array}\right] \quad \text { (12 planes) }} \\
& {\left[\begin{array}{cccc}
+ & + & 0 & 0 \\
+ & - & 0 & 0
\end{array}\right] \quad \text { (6 planes) }}
\end{aligned}
$$

Three different sets of principal angles occur: $(0, \pi / 2),(\pi / 4, \pi / 4)$, and $(\pi / 2, \pi / 2)$. Thus $d_{c}^{2}=1$ and $d_{g}^{2}=1.2337$. The automorphism group of this packing has structure $[3,4,3] .2$ and order 2304 . 

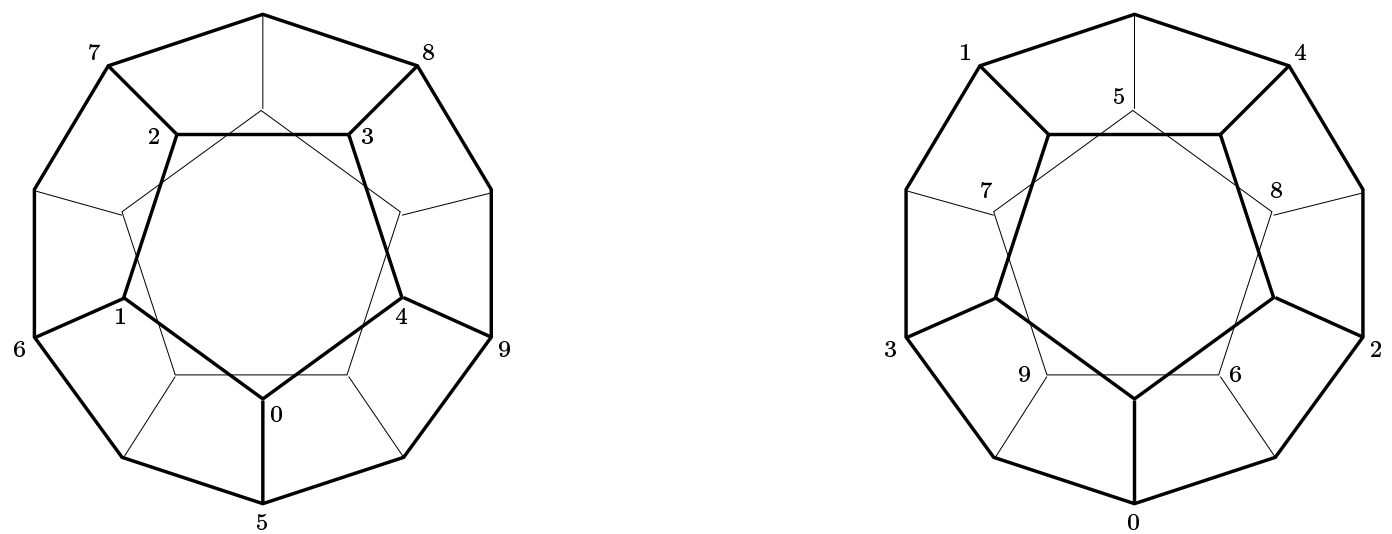

FIGURE 9. Matching of vertices of dodecahedron.

These 18 planes can also be described as the set of planes that meet the 24 -cell in one of its equatorial squares.

$N=48$ and $N=50$

Let $\mathcal{C}$ denote the set of vertices of a cube. Then the binocular codes for the 48- and 50-plane packings are respectively $(\mathcal{O} \times \mathcal{C}) \cup(\mathcal{C} \times \mathcal{O})$ and $(\mathcal{O} \times \mathcal{O}) \cup$ $(\mathcal{C} \times \mathcal{C})$. For both arrangements we have $d_{c}^{2}=\frac{2}{3}$ and $d_{g}^{2}=0.7576$. Both are believed to be optimal. (We mention the 48-plane packing because of its greater symmetry.)

\section{The Matching Problem}

In order to maximize the minimal chordal distance between the planes, the matching between the left and right codes should (from (4.1)) be chosen so as to minimize the maximal value of $\cos \psi \cos \varphi$. Stated informally, the matching should be such that if two points are close together on one of the spheres then the points to which they are matched should be far apart on the other sphere. In the case of the icosahedron, for example (see Figure 12), the matching sends adjacent vertices to nonadjacent vertices. There is a unique way to do this.

At this point we were tempted to see if any new record packings in $G(4,2)$ could be obtained by taking the $2 N$ antipodal points corresponding to a good $N$-line packing in $G(3,1)$, and matching them with themselves in an optimal way. However, it was not easy to see how to solve the matching problem. Fortunately David Applegate (personal communication) found that it could be reformulated as an integer programming problem, as follows.

Given an antipodal set $S=\left\{P_{1}, \ldots, P_{2 N}\right\} \subseteq S^{2}$, we wish to find a permutation $\sigma$ of $S$ with the property that $\sigma\left(-P_{i}\right)=-\sigma P_{i}$ for all $i$, and such that the minimal value of $1-\left(P_{i} \cdot P_{j}\right)\left(\sigma P_{i} \cdot \sigma P_{j}\right)$ over $i \neq j$ is maximized. If we do the maximization by binary search, we may define $\pi\left(P_{i}, P_{j}\right)=1$ if $\sigma P_{i}=$ $P_{j}$, or 0 otherwise, with the constraints $\pi\left(P_{i}, P_{j}\right)=$ $\pi\left(-P_{i},-P_{j}\right)$

$$
\begin{aligned}
& \sum_{j=1}^{2 N} \pi\left(P_{i}, P_{j}\right)=1 \quad \text { for } 1 \leq i \leq 2 N, \\
& \sum_{i=1}^{2 N} \pi\left(P_{i}, P_{j}\right)=1 \quad \text { for } 1 \leq j \leq 2 N,
\end{aligned}
$$

and $\pi\left(P_{i}, P_{j}\right)+\pi\left(P_{k}, P_{l}\right)<1$ for all $1 \leq i, j, k, l \leq$ $2 N$ such that $1-\left(P_{i} \cdot P_{k}\right)\left(P_{j} \cdot P_{l}\right)<M$. There is a feasible solution if and only if $d_{c}^{2} \geq M$.

Applegate kindly implemented this procedure, and used it to solve the matching problem for our best packings in $G(3,1)$ and for various polyhedra. Unfortunately no new records have yet been obtained by this method.

\section{Best Packings for the Geodesic Metric}

So far we have mostly discussed packings that attempt to maximize the minimal chordal distance 
between planes. We also computed packings for the geodesic distance, and we shall now describe some of them. In general, however, they are much less symmetric than the chordal-distance packings, especially for more than 16 planes.

For $N=4,5$ the solutions are subsets of the 6 plane packing. For $N=7$ the left code is a heptagonal antiprism and the right code is an equatorial 14-gon.

For $N=12$ the binocular code is given by the set $\{ \pm(l, r)\}$, where $l$ runs through the vertices of a regular tetrahedron and $r$ through those of an equatorial equilateral triangle.

For $N=16$ the left and right codes are the union of two similarly oriented square prisms. For larger $N$ the geodesic packings do not seem so interesting. For $N=18$, for example, the best packing has no nontrivial symmetries.

\section{Hamiltonian Paths}

One further question remains to be discussed, that of arranging the planes in a circuit in such a way that adjacent planes are close together, for the grand tour application. This turned out to be a much easier problem than finding the packings. We handled it in two different ways. For configurations such as the 48-plane arrangement, where there was an obvious notion of adjacency - in this case, defining two planes to be adjacent if the principal angles are $\pi / 4, \pi / 4-$ we represent the packing by a graph with nodes representing the planes, and look for a Hamiltonian cycle. In less regular cases, we convert the packing into a traveling salesman problem using chordal distance to define the distance between nodes, and look for a minimal length circuit. In both cases we were able to make use of the travelling salesman programs described in [Applegate et al. 1995], which can handle 100-node graphs without difficulty. In the public archive containing our results (see Electronic Availability before the references), the files with suffix .ham have been arranged in cycles in this way.

The above reformulation in terms of binocular codes applies only to $G(4,2)$; we knew from the beginning that this case would be special, since $G(4,2)$ is the only Grassmannian where the Riemannian metric is not unique [Leichtweiss 1961]. In the next section we describe a second reformulation that applies to the general case.

\section{PACKING n-PLANES IN HIGHER DIMENSIONS}

Three observations contributed to the second reformulation.

First, as can be seen in Table 2, for several examples of $N$-plane packings in $G(4,2)$ the largest value of $d_{c}^{2}$ that we could attain was $N /(N-1)$, and in every case this was an upper bound. Further experimentation with other packings in $G(m, n)$ for $m \leq 8$ led us to guess an upper bound of

$$
d_{c}^{2} \leq \frac{n(m-n)}{m} \frac{N}{N-1},
$$

which again we could achieve for some small values of $N$. The form of (5.1) was suggestive of the Rankin bound for spherical codes [Conway and Sloane 1996] or the Plotkin bound for binary codes [MacWilliams and Sloane 1977].

Secondly, investigation of the 18-plane chordaldistance packing revealed that, with respect to $d_{c}$, this packing has the structure of a regular orthoplex (or generalized octahedron) with 18 vertices. Combining this with the fact that the 10-plane packing formed a regular simplex, we had strong evidence that $G(4,2)$ should have an isometric embedding (with respect to $d_{c}$ ) into $\mathbb{R}^{9}$.

Finally, a computer program was therefore written to determine the lowest dimensions into which our library of packings in $G(m, n)$ could be isometrically embedded. More precisely, for a given set of $N$ planes in $G(m, n)$, we searched for the smallest dimension $D$ such that there are $N$ points in $\mathbb{R}^{D}$ whose Euclidean distances coincide with the chordal distances between our planes.

The results were a surprise: it appeared that $G(m, n)$ with chordal distance could be isometrically embedded into $\mathbb{R}^{D}$, for $D=\left(\begin{array}{c}m+1 \\ 2\end{array}\right)-1$, independent of $n$. Furthermore, the points representing 
elements of $G(m, n)$ were observed to lie on a sphere of radius $\sqrt{n(m-n) / 2 m}$ in $\mathbb{R}^{D}$.

Aided by discussions with Colin Mallows, we soon found an explanation: just associate to each $P \in G(m, n)$ the orthogonal projection map from $\mathbb{R}^{m}$ to $P$. If $A$ is a generator matrix for $P$ whose rows are orthogonal unit vectors, then the projection is represented by the matrix $\mathcal{P}=A^{t} A$, where ${ }^{t}$ denotes transposition. $\mathcal{P}$ is an $m \times m$ symmetric idempotent matrix, which is independent of the particular orthonormal generator matrix used to define it. Changing to a different coordinate frame in $\mathbb{R}^{m}$ has the effect of conjugating $\mathcal{P}$ by an element of $O(m)$. With the help of (2.1), we see that

$$
\text { trace } \mathcal{P}=n \text {. }
$$

Thus $\mathcal{P}$ lies in a space of dimension $\left(\begin{array}{c}m+1 \\ 2\end{array}\right)-1$.

Let $\|\cdot\|$ denote the $L_{2}$-norm of a matrix, that is,

$$
\|M\|=\sqrt{\sum_{i=1}^{m} \sum_{j=1}^{m} M_{i j}^{2}}=\sqrt{\operatorname{trace} M^{t} M} .
$$

if $M=\left(M_{i j}\right)$ with $1 \leq i, j \leq m$. For $P, Q \in$ $G(m, n)$, with orthonormal generator matrices $A$,
$B$, and principal angles $\theta_{1}, \ldots, \theta_{n}$, an elementary calculation using (2.1) and (2.2) shows that

$$
\begin{aligned}
d_{c}^{2}(P, Q) & =n-\left(\cos ^{2} \theta_{1}+\cdots+\cos ^{2} \theta_{n}\right) \\
& =n-\operatorname{trace} A^{t} A B^{t} B=\frac{1}{2}\|\mathcal{P}-Q\|^{2},
\end{aligned}
$$

where $\mathcal{P}, \mathcal{Q}$ are the corresponding projection matrices.

Defining the traceless part of $\mathcal{P}$ as $\overline{\mathcal{P}}=\mathcal{P}-$ $(n / m) I_{m}$, we have $\|\overline{\mathcal{P}}\|^{2}=n(m-n) / m$. We have thus established the following theorem.

Theorem 5.1. Let $D=\left(\begin{array}{c}m+1 \\ 2\end{array}\right)-1$. The representation of n-planes $P \in G(m, n)$ by their projection matrices $\overline{\mathcal{P}}$ gives an isometric embedding of $G(m, n)$ into a sphere of radius $\sqrt{n(m-n) / m}$ in $\mathbb{R}^{D}$, with $d_{c}(P, Q)=\frac{1}{\sqrt{2}}\|\mathcal{P}-\mathcal{Q}\|$.

Thus chordal distance between planes is $\frac{1}{\sqrt{2}}$ times the straight-line distance between the projection matrices (which explains our name for this metric). The geodesic distance between the planes is $\frac{1}{\sqrt{2}}$ times the geodesic distance between the projection matrices, measured along the sphere in $\mathbb{R}^{D}$.

Figure 10 attempts to display the embeddings of $G(m, 0), G(m, 1), \ldots, G(m, m)$ in $\mathbb{R}^{D+1}$. Since

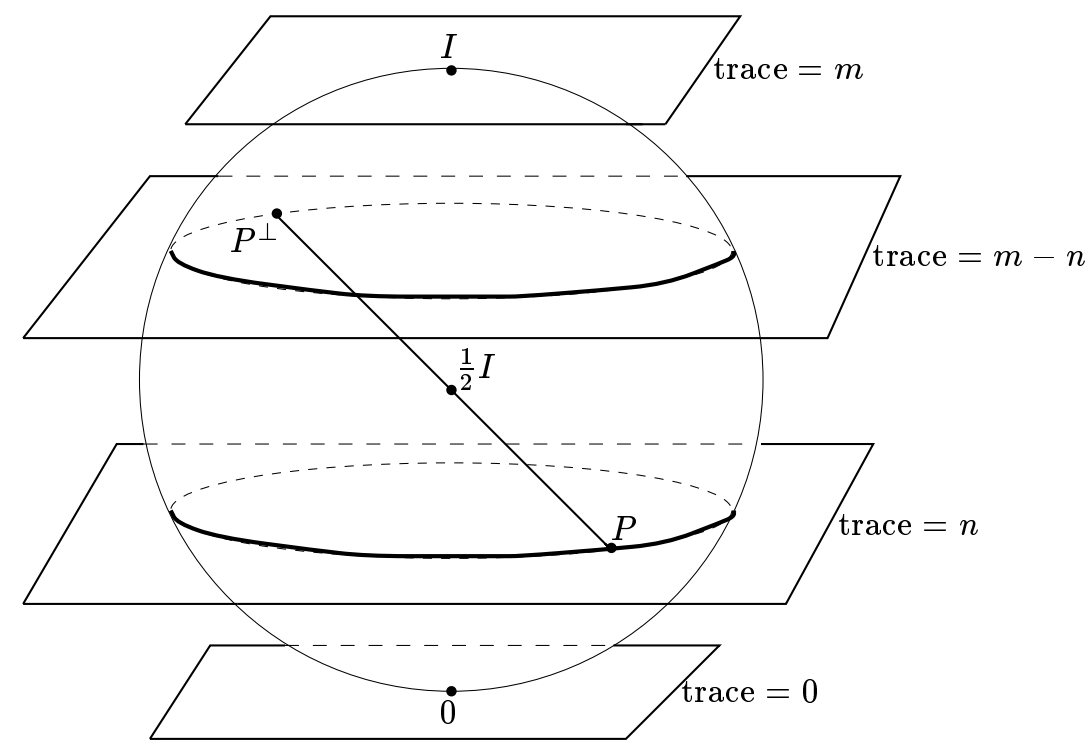

FIGURE 10. Embedding of $G(m, 0), \ldots, G(m, m)$ into large sphere of radius $\frac{1}{2} \sqrt{m}$ in Euclidean space of dimension $\frac{1}{2} m(m+1)$. The space $G(m, n)$ lies on sphere of radius $\sqrt{n(m-n) / m}$ in $\mathbb{R}^{D}$, where $D=\frac{1}{2}(m-1)(m+2)$. 
$\left\|\mathcal{P}-\frac{1}{2} I_{m}\right\|^{2}=\frac{1}{4} m$, all the planes lie on the large sphere, centered at $\frac{1}{2} I_{m}$, of radius $\frac{1}{2} \sqrt{m}$. The members of $G(m, n)$ lie on the intersection of the large sphere with the plane trace $\mathcal{P}=n$, which intersection is itself a sphere in $\mathbb{R}^{D}$ of radius $\sqrt{n(m-n) / m}$ centered at $\frac{n}{m} I_{m}$. A plane and its orthogonal complement are represented by antipodal points on the large sphere.

In contrast to this result, we briefly remark, without giving details, that there is no way to embed $G(m, n)$ into Euclidean space of any dimension so that the geodesic distance $d_{g}$ on $G(m, n)$ is represented by Euclidean distance in that space. Of course the Plücker embedding, in which members of $G(m, n)$ are represented by points in projective space of dimension $\left(\begin{array}{l}m \\ n\end{array}\right)-1$, also does not give a way to realize either $d_{c}$ or $d_{g}$ as Euclidean distance. (Nor does the Nash embedding theorem [Nash 1956].) Note also that the dimension of the Plücker embedding is in general much larger than the dimension of our embedding.

Since we have embedded $G(m, n)$ into a sphere of radius $\sqrt{n(m-n) / m}$ in $\mathbb{R}^{D}$, we can apply the Rankin bounds for spherical codes [Rankin 1955], and deduce:

Corollary 5.2 (The simplex bound). For a packing of $N$ planes in $G(m, n)$,

$$
d_{c}^{2} \leq \frac{n(m-n)}{m} \frac{N}{N-1} .
$$

Equality requires $N \leq D+1=\left(\begin{array}{c}m+1 \\ 2\end{array}\right)$, and occurs if and only if the $N$ points in $\mathbb{R}^{D}$ corresponding to the planes form a regular "equatorial" simplex.

Corollary 5.3 (The orthoplex bound). For $N>\left(\begin{array}{c}m+1 \\ 2\end{array}\right)$,

$$
d_{c}^{2} \leq \frac{n(m-n)}{m}
$$

Equality requires $N \leq 2 D=(m-1)(m+2)$, and occurs if the $N$ points form a subset of the $2 D$ vertices of a regular orthoplex. If $N=2 D$ this condition is also necessary.
Lemmons and Seidel ([Lemmens and Seidel 1973b], Theorem 3.6) give a bound for equi-isoclinic packings in $G(m, n)$ which agrees with (5.2); of course our bound is more general. The case $n=1$ of (5.2) is given in Theorem 3 of [Rosenfeld a].

We were happy to obtain confirmation of (5.1). Since $d_{c}^{2}$ can never exceed $n$, we can also write

$$
d_{c}^{2} \leq \min \left\{n, \frac{n(m-n)}{m} \frac{N}{N-1}\right\} .
$$

Corollaries 5.2 and 5.3 allow us to establish the optimality of many of our packings. In the range $m \leq 16, n \leq 3, N \leq 55$, there are over 750 cases which appear to meet (5.4) or (5.3), in the sense that the ratio of $d_{c}^{2}$ to the bound is greater than 0.9999999. Table 3 lists these cases for $n=2$ and $4 \leq m \leq 10$.

However, this is not as meaningful as it at first seems. Consider Table 4, which gives the ratio of $d_{c}^{2}$ to the simplex bound for packings of $N=$ $21, \ldots, 33$ planes in $G(10,2)$. From this table it seems very likely that there exists a 27 -plane pack-

\begin{tabular}{|rll|rll|}
\hline$m$ & $(5.4)$ & $(5.3)$ & $m$ & \multicolumn{1}{c|}{$(5.4)$} & $(5.3)$ \\
\hline 4 & $2-8,10$ & $11-18$ & 8 & $4-21,28$ & $37-44$ \\
5 & $4-11$ & 16,17 & 9 & $6-25$ & \\
6 & $3-14$ & 22,23 & 10 & $5-28$ & \\
7 & $6-18$ & 29 & & & \\
\hline
\end{tabular}

TABLE 3. Values of $N$ for which the packings of $N$ planes in $G(m, 2)$ appear to achieve the bounds (5.4) or (5.3).

\begin{tabular}{|cc|cc|}
\hline$N$ & ratio & $N$ & ratio \\
\hline 21 & 0.99999999999999 & 28 & 0.99999999921354 \\
22 & 1.00000000000000 & 29 & 0.99999470290071 \\
23 & 0.99999999999999 & 30 & 0.99998661084736 \\
24 & 0.99999999999999 & 31 & 0.99961159256647 \\
25 & 1.00000000000000 & 32 & 0.99909699728979 \\
26 & 0.99999999999999 & 33 & 0.99854979246655 \\
27 & 1.00000000000000 & & \\
\hline
\end{tabular}

TABLE 4. Ratio of $d_{c}^{2}$ to the bound of Corollary 5.2 for $N$ planes in $G(10,2)$, rounded to 14 decimal places. 
ing meeting the simplex bound, but certainly further investigation is needed to determine if there is a 28-plane simplex packing in the neighborhood of the computer's approximate solution. We have carried out such analyses in all the putative cases of equality for packing lines (see Sect. 6), for planes in $G(4,2)$ (as already discussed) and for 70 planes in $G(8,4)$ (see below). The other cases remain to be investigated.

In principle there is no difficulty in settling these questions. All the chordal distances between the planes are specified. So we could simply set up a (large) system of quartic equations with integer coefficients for the entries in the generator matrices, and ask if there is a real solution.

The chief difficulty in attempting to understand the computer-generated packings is that there are usually very large numbers of equally good solutions. The case of 18 planes in $G(4,2)$ is typical in this regard. There is one exceptionally pleasing solution (described in Section 4), in which only three different pairs of principal angles occur. However, it seems that there is a roughly 29-dimensional manifold of solutions, in which a typical solution, although still having $d_{c}^{2}=1$, has an apparently random set of principal angles. Our investigation of this question is not yet completed.

The case of subspaces of dimension $n$ in $\mathbb{R}^{2 n}$ or $\mathbb{R}^{2 n+1}$ is especially interesting. Table 5 summarizes the possible cases where the bounds can be achieved. It is known that the orthoplex bound cannot be achieved by 10 planes in $G(3,2)$, while the other cases are undecided. Our computer ex-

\begin{tabular}{|cccc|cccc|}
\hline$m$ & $n$ & $(5.4)$ & $(5.3)$ & $m$ & $n$ & $(5.4)$ & $(5.3)$ \\
\hline 2 & 1 & $3^{*}$ & $4^{*}$ & 6 & 3 & 21 & 40 \\
3 & 1 & $6^{*}$ & 10 & 7 & 3 & $28^{*}$ & 54 \\
4 & 2 & $10^{*}$ & $18^{*}$ & 8 & 4 & 36 & $70^{*}$ \\
5 & 2 & 15 & 28 & & & & \\
\hline
\end{tabular}

TABLE 5. Largest values of $N$ for which the packings of $N$ planes in $G(m, n)$ can achieve the bounds (5.4) or (5.3). An asterisk means the bound is achieved. periments strongly suggest that no set of 15 planes meets the simplex bound in $G(5,2)$.

On the other hand, it is possible to find packings of 70 planes in $G(8,4)$ meeting the bound $(5.3)$. With a considerable amount of effort (a long story, not told here), we determined several examples, of which the following is the most symmetrical. Let the coordinates be labeled $\infty, 0,1, \ldots, 6$, and take the two 4-planes generated by the vectors

$$
\{10000000,01000000,00100000,00001000\},
$$$$
\{11000000,00101000,00010001,00000110\} \text {. }
$$

From them we get 70 planes by negating any even number of coordinates, and by applying the coordinate permutations $(0123456),(\infty 0)(16)(23)(45)$, and $(124)(365)$. The principal angles are given by $(0,0, \pi / 2, \pi / 2),(\pi / 4, \pi / 4, \pi / 4, \pi / 4)$, or $(\pi / 2, \pi / 2$, $\pi / 2, \pi / 2$ ), so $d_{c}^{2}=2$ and $d_{g}^{2}=\pi^{2} / 4$ (this is not even a local optimum with respect to geodesic distance). The full group, which is transitive on the planes, is generated by the above operations and by the $8 \times 8$ Hadamard matrix

$$
\frac{1}{\sqrt{8}}\left[\begin{array}{c}
-------- \\
-+--+-++ \\
---+-+++ \\
--+-+++- \\
-+-+++-- \\
--+++--+ \\
-+++--+- \\
-++--+-+
\end{array}\right]
$$

where - means -1 and + means +1 . It has shape $2^{8} \mathcal{A}_{8}$ and order $5160960\left(\mathcal{A}_{n}\right.$ denotes an alternating group of degree $n$ ).

A set of 28 planes in $G(7,3)$ meeting the simplex bound can be obtained as follows. Label the coordinates $0, \ldots, 6$, and let $v_{r}^{ \pm}$have components 1 at $2^{r} \bmod 7, \pm \sqrt{2}$ at $3 \cdot 2^{r} \bmod 7$, and 0 's elsewhere, for $r=0,1,2$. Then the vectors $v_{0}^{ \pm}, v_{1}^{ \pm}$, $v_{2}^{ \pm}$(where the product of signs is even) span four planes, and the full set of 28 is found by cycling the seven coordinates.

(Note added April 1996. P. W. Shor and Sloane have recently discovered that the packing of 70 planes in $G(8,4)$ can be generalized to a packing 
of $m^{2}+m-2$ planes in $G\left(m, \frac{1}{2} m\right)$ meeting the orthoplex bound, whenever $m \geq 2$ is a power of 2 ; and that the packing of 28 planes in $G(7,3)$ can be generalized to a packing of $\frac{1}{2} p(p+1)$ planes in $G\left(p, \frac{1}{2}(p-1)\right)$ meeting the simplex bound, whenever $p$ is a prime which is either 3 or congruent to -1 modulo 8 . These constructions will be described elsewhere.)

On the other hand, in spite of much effort, we have not been able to find a set of 40 planes in $G(6,3)$ that meet the bound. If this were possible, we would obtain $d_{c}^{2}=1.5$, whereas the best we have been able to achieve is 1.49977. We can get 1.5 with $N=34$ planes, and 1.49977 with $35 \leq N \leq 40$, but only 1.4297 with 41 planes, which suggests that 1.5 might be possible with 40 planes. The discussion given earlier shows that this question could in principle be settled by seeing if a certain set of quartic equations with integer coefficients has a real solution. One formulation leads to 500 quartic equations in 360 unknowns, so this approach seems hopeless at the present time.

It is not difficult to invent ways to construct sets of subspaces. Several examples are given below. However, many promising ideas have proved useless when confronted with the results found by our program. For this reason we have included two extensive tables of the best chordal distances that we have found. Table 6 gives $d_{c}^{2}$ for packings in $G(m, 2)$, with $m \leq 10$. This will serve as a standard against which readers can test their own constructions.

\section{General Constructions}

We now proceed to list some promising general constructions.

\begin{tabular}{|c|c|c|c|c|c|c|c|c|c|c|c|c|}
\hline$N$ & $=4$ & 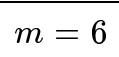 & & 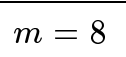 & $n$ & $=1$ & $N m=4 r$ & & & & & \\
\hline 3 & & & & & & & 270.80561. & & & & & \\
\hline 4 & & 1.7778 & & & & & $\begin{array}{lll}8 & 0.8005 & 1.0989\end{array}$ & & & & & 6593 \\
\hline 5 & 5001.5000 & 1.6667 & & 8750 & 1.9375 & 00 & 891.0937 & & & & & 6571 \\
\hline 6 & 001.4400 & & & & 8667 & 00 & 300. & & & & & 6552 \\
\hline 7 & & & & & & & & & & & & 527 \\
\hline 8 & & & & & & 86 & 011. & & & & & 01 \\
\hline 9 & & & & & & & & & & & & 76 \\
\hline 10 & & & & & & & & & & & & 148 \\
\hline 11 & & & & & & & & & & & & \\
\hline 12 & & & & & & & & & & & & 114 \\
\hline 13 & & & & & & & & & & & & \\
\hline 14 & & & & & & & 981. & & & & & 1.6334 \\
\hline 15 & & & & & & & 551. & & & & & 1.6305 \\
\hline 16 & & & & & & & & & & & & 289 \\
\hline 17 & & & & & & & & & & & & 242 \\
\hline 18 & & & & & & & 81. & & & & & 213 \\
\hline 19 & & & & & 120 & & & & & & & 192 \\
\hline 20 & & & & & 6374 & & 59 & & & & & 1.6174 \\
\hline 21 & 1543 & & & & 1.6333 & 1.6800 & 991. & & & & 570 & 1.6160 \\
\hline 22 & $0.8629 \quad 1.1419$ & 1.3333 & 1.4912 & 1.5714 & 1.6296 & 1.6762 & 931.0078 & 1.1941 & 321 & 321 & 5480 & 1.6099 \\
\hline 23 & $0.8451 \quad 1.1332$ & 1.3333 & & 1.5680 & 1.6263 & 1.6727 & 67321.0042 & & 1.3265 & & 5412 & 1.6072 \\
\hline 24 & 0.83721 .1251 & 1.3326 & 1.4790 & 1.5638 & 1.6232 & 1.6696 & $\begin{array}{llll}48 & 0.6667 & 1.0001\end{array}$ & 1.1907 & 1.3232 & 1.4490 & .5353 & 1.6045 \\
\hline 25 & $0.8275 \quad 1.1178$ & 1.3229 & & & 1.6204 & 1.6667 & $\begin{array}{llll}49 & 0.6667 & 0.9960\end{array}$ & & & & 1.5304 & 1.6019 \\
\hline 26 & $0.8144 \quad 1.1113$ & 1.3151 & 1.4666 & 1.5556 & 1.6177 & 1.6640 & $\begin{array}{llll}50 & 0.6667 & 0.9910\end{array}$ & 1.1841 & 1.3150 & 1.4370 & 1.5257 & 1.6003 \\
\hline
\end{tabular}

TABLE 6. Values of $d_{c}^{2}$ for best packings found of $N \leq 50$ planes in $G(m, 2)$. 
(i) A skew-symmetric conference matrix of order $4 a$ yields a set of $4 a$ unit vectors in $\mathbb{C}^{2 a}$ with Hermitean inner products $\pm i / \sqrt{4 a-1}$ [Delsarte et al. 1975, Example 5.8], hence a set of $4 a$ planes in $G(4 a, 2)$ with $d_{c}^{2}=4(2 a-1) /(4 a-1)$ that meet the simplex bound. (For a list of the known orders of such matrices, see [Seberry and Yamada 1992, Table 7.1].)

(ii) Use the planes defined by the $n$-faces of a regular $m$-dimensional polytope, or the Voronoi or Delaunay cells of a lattice, etc. The initial results from this idea have been disappointing. For example, the 96 two-dimensional faces of the 24 -cell in $\mathbb{R}^{4}$ define 16 different planes, forming a packing with $d_{c}^{2}=\frac{8}{9}$, inferior to the best such packing as seen in Table 6 .

(iii) Use the minimal vectors in a complex or quaternionic lattice in order to obtain packings in $G(2 a, 2)$ or $G(4 a, 4)$. For example, the 54 minimal vectors of the lattice $E_{6}^{*}$, regarded as a three-dimensional lattice over the Eisenstein integers [Conway and Sloane 1996, p. 127], produce a packing of nine planes in $G(6,2)$ that meets the simplex bound.

(iv) Restrict the search to generator matrices of 0 's and 1's (as in (5.5)) or +1 ' and -1 's, or even to rows that are blocks in some combinatorial design, or vectors in some error-correcting code (see the example in Section 6).

(v) Let $C$ be an error-correcting code of length $m$ over $\mathrm{GF}\left(2^{n}\right)$, for example a Reed-Solomon code [MacWilliams and Sloane 1977]. If we see $\operatorname{GF}\left(2^{n}\right)$ as a vector space of dimension $n$ over GF(2), each codeword yields an $n \times m$ matrix whose elements we may take to be +1 's and -1 's. After discarding those of rank less than $n$, and weeding out duplicates, we obtain a packing in $G(m, n)$. For example, the hexacode [Conway and Sloane 1996, p. 82], a code of length 6 over $\mathrm{GF}(4)$ containing 64 codewords, produces 28 distinct planes in $G(6,2)$ with $d_{c}^{2}=3 / 4$. Unfortunately Table 7 shows that the record is 1.2973 . (vi) Choose a group $G$ with an $m$-dimensional representation, and a subgroup $H$ of index $N$ with an $n-$ dimensional irreducible representation. Find an $n$ dimensional subspace $V \subseteq \mathbb{R}^{m}$ invariant under $H$, and take its orbit under $G$. Many of the conference matrix constructions of line-packings described in Section 6 are of this type (using $G=L_{2}(q)$ ).

In some cases we have also used the optimizer to search for packings with a specified group. The following is a packing of 28 planes in $G(8,2)$ meeting the simplex bound that is an abstraction of a configuration found by the computer when searching for packings in $\mathbb{R}^{8}$ invariant under the permutation $(0)(1,2,3,4,5,6,7)$. Let $R: \mathbb{C}^{4} \rightarrow \mathbb{R}^{8}$ map $\left(v_{1}, v_{2}, v_{3}, v_{4}\right)$ to $\left(\operatorname{Re} v_{1}, \operatorname{Im} v_{1}, \operatorname{Re} v_{2}, \ldots, \operatorname{Im} v_{4}\right)$, and let $\alpha=e^{2 \pi i / 7}$. The 28 planes are spanned by the following pairs of vectors, where $k$ ranges from 0 to 6 :

$$
\begin{array}{ll}
R\left(0, \alpha^{k}, \alpha^{2 k}, \alpha^{4 k}\right), & R\left(0, i \alpha^{k}, i \alpha^{2 k}, i \alpha^{4 k}\right), \\
R\left(1,0, \alpha^{2 k}, \alpha^{4 k}\right), & R\left(i, 0, i \alpha^{2 k},-i \alpha^{4 k}\right), \\
R\left(1, \alpha^{k}, 0, \alpha^{4 k}\right), & R\left(i,-i \alpha^{k}, 0, i \alpha^{4 k}\right), \\
R\left(1, \alpha^{k}, \alpha^{2 k}, 0\right), & R\left(i, i \alpha^{k},-i \alpha^{2 k}, 0\right) .
\end{array}
$$

The pattern of zeros and signs here suggests the tetracode [Conway and Sloane 1996, p. 81].

\section{An Application: Apportioning Randomness}

Here is a potential application of packings in Grassmanian spaces for use in apportioning randomness, in the sense of producing large numbers of approximately random points from a few genuinely random numbers. We illustrate using the example of 70 planes in $G(8,4)$ described above. Let $A_{1}, \ldots, A_{70}$ be generator matrices for them. Suppose an exclusive resort wishes to distribute random points in $\mathbb{R}^{4}$ to its 70 guests, perhaps for use as garage door keys. Let $x=\left(x_{1}, \ldots, x_{8}\right)$ be a vector of eight independent Gaussian random variates. Then the hotel would assign $y_{i}=A_{i} x^{t}$ to its $i$-th guest. By maximizing the chordal distance between the planes we have minimized the correlation between the $y_{i}$. 


\begin{tabular}{|c|c|c|c|c|c|c|c|c|c|c|c|c|c|c|c|}
\hline$N$ & $m=3$ & $m=4$ & $m=5$ & $m=6$ & $m=7$ & $m=8$ & $m=9$ & $N$ & $m=3$ & $m=4$ & $m=5$ & $m=6$ & $m=7$ & $m=8$ & $m=9$ \\
\hline 3 & & & & & & 90.0000 & 90.0000 & 27 & & 43.5530 & & & & 70.5392 & 71.6650 \\
\hline 4 & & & .0000 & 90.0000 & 90.0000 & 90.0000 & 90.0000 & 28 & 8473 & 43.1566 & 53.2602 & 60.5276 & $70.5288^{b}$ & 70.5322 & 71.5794 \\
\hline 5 & $63.4349^{f}$ & $75.5225^{\mathrm{d}}$ & & 90.0000 & 90.0000 & 90.0000 & 90.0000 & 29 & .5244 & 42.6675 & 53.0180 & 60.1344 & 66.7780 & 70.5288 & 71.5175 \\
\hline 6 & & & & & & 0.0000 & 90.0000 & 30 & 9983 & 42.2651 & 52.7812 & 60.0213 & 65.7563 & 70.5288 & 71.5175 \\
\hline 7 & $54.7356^{\mathrm{f}}$ & & & $80.4059^{\mathrm{d}}$ & & & 90.0000 & 31 & .4987 & 42.0188 & 52.4120 & & & & 70.8508 \\
\hline 8 & & & & & 017 & & 000 & 32 & & & & & & & 7437 \\
\hline 9 & & & & & & $82.8192^{\mathrm{d}}$ & & 33 & & & & & & & 940 \\
\hline 10 & 46.674 & & $.5288^{\mathrm{b}}$ & 73.6935 & 76.3454 & 79.4704 & $83.6206^{\mathrm{d}}$ & 34 & 25.2567 & 40.9427 & 51.8537 & 60.0000 & 64.6231 & 69.1688 & 70.6512 \\
\hline 11 & & & & & & & 80.6204 & 35 & & & & & & & 6337 \\
\hline 12 & 41. & 60. & 213 & 71.5651 & 74.1734 & 76.6050 & 79.4704 & 36 & 24.5758 & 40.6325 & 51.8273 & $60.0000^{2}$ & 64.6231 & $69.0752^{\mathrm{e}}$ & 70.5864 \\
\hline 13 & & .4646 & 319 & 70.5288 & 73.8979 & 1645 & 422 & 37 & .2859 & 40.4486 & .8273 & 6885 & 62.3797 & 827 & 70.5695 \\
\hline 14 & & & 241 & .5288 & 73.8 & & 382 & 38 & & 419 & & & & & 5571 \\
\hline 15 & & & .5302 & 70.5288 & 71.5 & & 006 & 39 & & & 50.0611 & 8357 & & & 443 \\
\hline 16 & $37.3774^{\mathrm{f}}$ & 51.8273 & $.4349^{\mathrm{e}}$ & $70.5288^{b}$ & 70.9861 & 74.1005 & 9638 & 40 & 23.3293 & $39.0236^{\mathrm{e}}$ & 49.5978 & 56.0495 & 61.3792 & 66.3 & 70.5288 \\
\hline 17 & 53 & & 2551 & 68.1088 & & 1371 & 638 & 41 & 915 & 346 & & 8202 & & & 5288 \\
\hline 18 & 3 & 4577 & & & & & $638^{c}$ & 42 & & & & & & & 5288 \\
\hline 19 & & & & & & & & 43 & & & & & & & 288 \\
\hline 20 & 7071 & 49.2329 & & & 70.5288 & 1.6706 & 74.2278 & 44 & 2.2012 & 37.3474 & 48.0955 & 55.1259 & 60.3623 & 65.2422 & 70.5288 \\
\hline 21 & & & & & & & & 45 & & & & & & & 288 \\
\hline 22 & 3 & & & & & & & 46 & & & & & & 64. & 5288 \\
\hline 23 & 30.5062 & & & & & & & 47 & & & & & & & 70.5288 \\
\hline 24 & 30.1628 & 46.0478 & הפרת & 63.6122 & 70.5288 & 70.6027 & 72.6547 & 48 & 21.46 & 36.35 & 46.7 & 54.59 & 60.0 & 63.8846 & $70.5288^{b}$ \\
\hline 25 & 29.2486 & & & & & & & 49 & & & & & & 63.4849 & 68.0498 \\
\hline 26 & 28.7126 & 44.3536 & 54.2116 & 61.7377 & 70.5288 & 70.5432 & 72.1763 & 50 & 20.8922 & 36.0754 & 46.1609 & 54.3191 & $60.0000^{\mathrm{a}}$ & 63.1527 & 67.7426 \\
\hline
\end{tabular}

TABLE 7. Maximal angular separation found for $N \leq 50$ lines in $G(m, 1)$. Superscript key: "a" and "b" indicate constructions from sphere packings, "c" from conference matrices, and "d" from diplo-simplices; "e" indicates a packing described below, and "f" one described in Section 3.

\section{PACKING LINES IN HIGHER DIMENSIONS}

Table 7 shows the maximal angular separation we have found for packings of $N \leq 50$ lines in $G(m, 1)$ for $m \leq 9$.

Table 8 shows the packings of $N$ lines in $G(m, 1)$, for $m \leq 10$, that achieve either the simplex or orthoplex bounds. Most of them are described below.

\begin{tabular}{|rl|rl|}
\hline$m$ & \multicolumn{1}{c|}{$N$} & $m$ & \multicolumn{1}{c|}{$N$} \\
\hline 3 & $3,4,6,7$ & 7 & $7,8,14,28$ \\
4 & $4,5,11,12$ & 8 & 8,9 \\
5 & $5,6,10,16$ & 9 & $9,10,18,46-48$ \\
6 & $6,7,16,22$ & 10 & $10,11,16$ \\
\hline
\end{tabular}

TABLE 8. Values of $N$ for which the packings of $N$ planes in $G(m, 1)$ appear to achieve either the simplex or orthoplex bounds.
As mentioned in Section 3, the best packing of 5 lines in $G(3,1)$ is a subset of the best packing of 6 lines. Table 4 shows similar phenomena in higher dimensions. For example, the putatively best packing of 48 lines in $G(9,1)$ is so good that we cannot do better even when up through 8 lines are omitted from it.

In the rest of this section we discuss some of the entries in Table 7 that have the largest symmetry groups.

\section{Constructions from Lattices}

Let $P_{1}, \ldots, P_{a}$ be mutually touching spheres in a $d$-dimensional lattice packing. If there are $2 N$ further spheres in the packing, each of which touches all of $P_{1}, \ldots, P_{a}$, their centers form a $(d-a+1)$ dimensional antipodal spherical code with angular 
separation $\operatorname{arcsec}(a+1)$ [Conway and Sloane 1996, p. 340, Theorem 1].

In particular, the entries with a superscript "a" in Table 7 are obtained from the centers of spheres that touch one sphere in the lattices $D_{4}, D_{5}, E_{6}$ and $E_{7}$. The entries with a superscript "b" are obtained from spheres that touch two spheres in the lattices $D_{4}, D_{5}, E_{6}, E_{7}, E_{8}, \Lambda_{9}$ and the nonlattice packing $P_{10 b}$ [Conway and Sloane 1996, Chapter 1, Table 1.2]. We remark the set of 28 lines in $G(7,1)$ with angle $\arccos \frac{1}{3}$ obtained in this way from $E_{8}$ is known to be unique: see [Conway and Sloane 1996, Chapter 14, Theorem 12]. This configuration of lines is derived in a different way in [Lint and Seidel 1966].

It is interesting to compare Table 7 with the table of maximal sets of equiangular lines given in Lemmens and Seidel [Lemmens and Seidel 1973a] and Seidel [Seidel 1994]. Some arrangements appear in both tables, for example the set of 28 lines in $G(7,1)$ just mentioned. On the other hand the difference between the tables can be seen in dimension 8 . The maximal set of equiangular lines that exists in $G(8,1)$ has size 28 , with angle $\operatorname{arcsec} 3$ ([Lemmens and Seidel 1973a], Theorem 4.6). However, Table 7 shows that there is a set of 28 lines (not equiangular) in $G(8,1)$ with minimal angle $\operatorname{arcsec} 3.000511 \ldots$. . In view of the Lemmons-Seidel result (which uses the fact that in an $m$-dimensional equiangular arrangement of $N$ lines with $N \geq 2 m$ the secant must be an odd integer) our set of 28 lines cannot be perturbed to give an equiangular set without decreasing the minimal angle.

\section{Constructions from Conference Matrices}

It follows from [Lint and Seidel 1966, Theorem 6.3] that if a symmetric conference matrix of order $q+1$ $\equiv 2(\bmod 4)$ exists then there is an arrangement of $q+1$ equiangular lines in $\mathbb{R}^{m}, m=\frac{1}{2}(q+1)$, with $d_{c}^{2}=(q-1) / q$, meeting the simplex bound. The corresponding entries have the superscript "c" in the table. Our program was able to find these packings for every prime power $q$ of this form below 100 except for 49 and 81 .

\section{Constructions from Diplo-Simplices}

The entries with a superscript " $\mathrm{d}$ " are obtained by using all the vectors of shape $\pm c\left(n^{1},(-1)^{n}\right)$, where $c=1 / \sqrt{n(n+1)}$. In the notation of [Conway and Sloane 1996, Chap. 4], they are the minimal vectors in the translates of the root lattice $A_{n}$ by the glue vectors [1] and [-1]. They form the vertices of a diplo-simplex [Conway and Sloane 1991], and have automorphism group $2 \times \mathcal{A}_{n+1}$. These packings also meet the simplex bound.

\section{Constructions from Codes}

Let $C$ be a binary code of length $m$, size $M$ and minimal distance $d$ which is closed under complementation. Writing the codewords as vectors of \pm 1 's, we obtain a packing of $\frac{1}{2} M$ lines in $G(m, 1)$ with $d_{c}^{2}=4 d(m-d) / m^{2}$. For example, a shortened Hamming code of length 10 with $M=32$, $d=4$ gives a packing of 16 lines in $G(10,1)$ meeting the simplex bound. This construction provides a rich supply of good packings. The NordstromRobinson code [Forney et al. 1993; Hammons et al. 1994], for example, yields a packing of 128 lines in $G(16,1)$ with $d_{c}^{2}=\frac{15}{16}$.

\section{0 lines in $\mathrm{G}(4,1)$}

The 80 antipodal points are given by $\left(\xi^{\mu+1 / 2}, 0\right)$, $\left(0, \xi^{\nu+1 / 2}\right),\left(a \xi^{2 \mu}, b \xi^{2 \nu+1}\right),\left(a \xi^{2 \mu+1}, b \xi^{2 \nu}\right),\left(b \xi^{2 \mu}, a \xi^{2 \nu}\right)$, $\left(b \xi^{2 \mu+1}, a \xi^{2 \nu+1}\right)$, where $\xi=e^{2 \pi i / 8}, \mu, \nu=0, \ldots, 3$, $a=2^{-1 / 4}, b=\sqrt{1-a^{2}}$. The group of these 80 points is the group $\frac{1}{2}\left(\mathcal{D}_{16}+\mathcal{D}_{16}\right) \cdot 2$ of order 256 (see [Conway and Sloane a]). Here $\mathcal{D}_{m}$ denotes a dihedral group of order $m$.

\section{2 lines in $G(6,1)$}

We use the vertices of a hemi-cube (an alternative way to describe the 16-line packing) together with the six coordinate axes.

\section{3 and 64 lines in $G(7,1)$}

The putatively best packing of 63 lines in $G(7,1)$ (just beyond the range of Table 7 ) has angular separation $60^{\circ}$, and is formed from the 126 minimal 
vectors of the lattice $E_{7}$. The automorphism group of this set of 126 points is the Weyl group $\mathcal{W}\left(E_{7}\right)$, of order $2^{10} \cdot 3^{4} \cdot 5 \cdot 7$. Compare [Conway and Sloane 1996, Chapter 4, § 8.3].

The best packing found of 64 lines in $G(7,1)$ also has an unusually large group, of order $2\left|\mathcal{W}\left(E_{6}\right)\right|=$ $2^{8} \cdot 3^{4} \cdot 5$. This packing can be obtained as follows. The largest gaps between the packing of 63 lines occur in the directions of the minimal vectors of the dual lattice $E_{7}^{*}$. Let $u$ be such a vector, with $u=\frac{1}{2} v_{6}, v_{6} \in E_{7}, v_{6} \cdot v_{6}=6$. Taking a coordinate frame in which the last coordinate is in the $v_{6}$ direction, we obtain the minimal vectors of $E_{7}$ in the form $(w, 0), w \in E_{6}, w \cdot w=2$ (72 vectors), and $(x, \pm \sqrt{2 / 3}), x \in E_{6}^{*}, x \cdot x=\frac{4}{3}$ (54 vectors). We now adjoin $\pm \sqrt{6}$ and rescale by multiplying the last coordinate by $\sqrt{3 / 4}$, obtaining our final configuration of vectors $(0, \pm 3 / \sqrt{2}),(w, 0)$, $(x, \pm 1 / \sqrt{2})$, which is isomorphic to what the computer found. The rescaling has compressed the $E_{7}$ lattice in the $v_{6}$ direction until the angle between $v_{6}$ and the $(x, 1 / \sqrt{2})$ vectors is the same as the minimal angle between the $(x, 1 / \sqrt{2})$ and $(w, 0)$ vectors. This angle, the minimal angle in the packing, is $\arccos \sqrt{3 / 11}=58.5178^{\circ}$.

\section{6 lines in $G(8,1)$}

The 72 antipodal points consist of all permutations of the two vectors $c\left(7^{2},-2^{7}\right)$ and $c\left(-7^{2}, 2^{6}\right)$, where $c=1 / \sqrt{126}$. These are the minimal vectors in the translates of the $A_{8}$ lattice by the glue vectors [2] and $[-2]$.

The configuration of 16 lines in $G(5,1)$ is similarly obtained from the translates of $A_{5}$ by [1], [3] and [5].

\section{9 lines in $\mathrm{G}(12,1)$}

Take the 13 lines of a projective plane of order 3, described by vectors of four 1's and nine 0's, and replace two of the 1's by -1's in all possible ways, obtaining 78 vectors in $\mathbb{R}^{12}$ with angle $\operatorname{arcsec} 4$. The group is $2 \times L_{3}(3)$, of order $2^{5} 3^{3} 13$.
If instead we replace any odd number of the 1's by -1 's, we obtain a putatively optimal packing of 52 lines in $G(13,1)$, with angle arcsec 4 and group $2 \times L_{3}(3) \cdot 2$ of order $2^{6} 3^{3} 13$.

As in all these examples, we were amazed that the program was able to discover such beautiful configurations.

\section{ACKNOWLEDGEMENTS}

We are grateful to Daniel Asimov, Andreas Buja, Dianne Cook, Simon Kochen, Warren Smith, and especially Colin Mallows for helpful discussions, and to David Applegate for help in solving the matching problem described in Section 4.

\section{ELECTRONIC AVAILABILITY}

The results of our computations are available in the netlib archive, at the address http://netlib.att.com/ math/sloane/grass.

\section{REFERENCES}

[Anderson 1984] T. W. Anderson, An Introduction to Multivariate Statistical Analysis, Wiley, New York, 2nd ed., 1984, Chap. 12.

[Applegate et al. 1995] D. Applegate, R. Bixby, V. Chvátal and W. Cook, "Finding cuts in the TSP (a preliminary report)", DIMACS Technical Report 9505, Rutgers University, 1995.

[Asimov 1985] D. Asimov, "The Grand Tour: a tool for viewing multidimensional data", SIAM J. Sci. Stat. Comput. 6 (1985), 128-143.

[Björck and Golub 1973] A. Björck and G. H. Golub, "Numerical methods for computing angles between linear subspaces", Math. Comp. 27 (1973), 579-594.

[Buja and Asimov 1986] A. Buja and D. Asimov, "Grand Tour methods: an outline", pp. 63-67 in Computer Science and Statistics, 17th Symposium on The Interface, Lexington, KY, 1985 (edited by D. M. Allen), Elsevier, Amsterdam, 1986.

[Chow 1949] W.-L. Chow, "On the geometry of algebraic homogeneous spaces", Annals Math. 50 (1949), 32-67. 
[Conway and Kochen] J. H. Conway and S. Kochen, Geometry and quantum logic, in preparation.

[Conway and Sloane 1991] J. H. Conway and N. J. A. Sloane, "The cell structures of certain lattices", pp. 71-107 in Miscellanea mathematica (edited by P. Hilton, et al.), Springer, New York, 1991.

[Conway and Sloane 1996] J. H. Conway and N. J. A. Sloane, Sphere Packings, Lattices and Groups, 2nd ed., Springer, New York, 1993.

[Conway and Sloane a] J. H. Conway and N. J. A. Sloane, "The four-dimensional point groups-a revised enumeration", in preparation.

[Coxeter 1950] H. S. M. Coxeter, "Self-dual configurations and regular graphs", Bull. Amer. Math. Soc. 56 (1950), 413-455.

[Coxeter 1974] H. S. M. Coxeter, "Polyhedral numbers", pp. 25-35 in For Dirk Struik (edited by R. S. Cohen et al.), Reidel, Dordrecht, 1974.

[Coxeter and Moser 1980] H. S. M. Coxeter and W. O. J. Moser, Generators and Relations for Discrete Groups, 4th ed., Springer, New York, 1980.

[Delsarte et al. 1975] P. Delsarte, J. M. Goethals and J. J. Seidel, "Bounds for systems of lines, and Jacobi polynomials", Philips Research Reports 30 (1975), 91-105.

[Du Val 1964] P. Du Val, Homographies, Quaternions and Rotations, Oxford Univ. Press, 1964.

[Fejes Tóth 1965] L. Fejes Tóth, "Distribution of points in the elliptic plane", Acta Math. Acad. Sci. Hungar. 16 (1965), 437-440.

[Forney et al. 1993] G. D. Forney, Jr., N. J. A. Sloane, and M. D. Trott, "The Nordstrom-Robinson code is the binary image of the octacode", pp. 19-26 in Coding and Quantization, DIMACS/IEEE, 1992 (edited by R. Calderbank, et al.), Amer. Math. Soc., Providence, 1993.

[MacKenzie and Morgan 1995] H. Gluck, D. MacKenzie and F. Morgan, "Volume-minimizing cycles in Grassmann manifolds", Duke Math J. 79 (1995), 335-404.

[Golub and Van Loan 1989] G. H. Golub and C. F. Van Loan, Matrix Computations, 2nd ed., Johns Hopkins Univ. Press, Baltimore, 1989.
[Griffiths and Harris 1978] P. Griffiths and J. Harris, Principles of Algebraic Geometry, Wiley, New York, 1978

[Hammons et al. 1994] A. R. Hammons, Jr., P. V. Kumar, A. R. Calderbank, N. J. A. Sloane, and P. Solé, "The $\mathbb{Z}_{4}$-Linearity of Kerdock, Preparata, Goethals and Related Codes", IEEE Trans. Information Theory 40 (1994), 301-319.

[Hardin and Sloane 1992] R. H. Hardin and N. J. A. Sloane, "Operating manual for Gosset: A generalpurpose program for constructing experimental designs", 2nd ed., Statistics Research Report 106, AT\&T Bell Laboratories, 1992; also DIMACS Technical Report 93-51, Rutgers University, 1993.

[Hardin and Sloane 1993] R. H. Hardin and N. J. A. Sloane, "A new approach to the construction of optimal designs", J. Statistical Planning and Inference 37 (1993), 339-369.

[Hardin et al. 1993-95] R. H. Hardin, N. J. A. Sloane and W. D. Smith, Tables of spherical codes in dimensions 3, 4, 5, http://netlib.att.com/math/ sloane/packings. The tables are based on our own calculations, with additional packings provided by D. A. Kottwitz and J. Buddenhagen, and are believed to contain the best packings known with $N \leq 130$ points in dimensions 3,4 and 5 .

[Hardin et al. a] R. H. Hardin, N. J. A. Sloane, and W. D. Smith, Spherical Codes, in preparation.

[Hooke and Jeeves 1961] R. Hooke and T. A. Jeeves, " 'Direct search' solution of numerical and statistical problems", J. Assoc. Comput. Math. 8 (1961), 212229.

[Hudson 1905] R. W. H. T. Hudson, Kummer's Quartic Surface, Cambridge Univ. Press, 1905.

[James and Constantine 1974] A. T. James and A. G. Constantine, "Generalized Jacobi polynomials as spherical functions of the Grassmann manifold", Proc. London Math. Soc. 29 (1974), 174-192.

[Leichtweiss 1961] K. Leichtweiss, "Zur Riemannschen Geometrie in Grassmannschen Mannigfaltigkeiten", Math. Zeit. 76 (1961), 334-366.

[Lemmens and Seidel 1973a] P. W. H. Lemmens and J. J. Seidel, "Equiangular lines", J. Algebra 24 (1973), 494-512. 
[Lemmens and Seidel 1973b] P. W. H. Lemmens and J. J. Seidel, "Equi-isoclinic subspaces of Euclidean space", Konink. Ned. Akad. Wet. A76 (1973), 98107.

[Lint and Seidel 1966] J. H. van Lint and J. J. Seidel, "Equilateral point sets in elliptic geometry", Proc. Kon. Nederl. Akad. Wet. A69 (1966), 335-348.

[MacWilliams and Sloane 1977] F. J. MacWilliams and N. J. A. Sloane, The Theory of Error-Correcting Codes, Elsevier, Amsterdam, 1977.

[Nash 1956] J. Nash, "The imbedding problem for Riemannian manifolds", Annals Math. 63 (1956), $20-63$.

[Rankin 1955] R. A. Rankin, "The closest packing of spherical caps in $n$ dimensions", Proc. Glasgow Math. Assoc. 2 (1955), 139-144.

[Rosenfeld 1993] M. Rosenfeld, Problem No. 10293, Amer. Math. Monthly 100 (1993), 291.

[Rosenfeld 1994] M. Rosenfeld, "How wide can you spread your chopsticks?" Congressus Numerantium 102 (1994), 29-31.

[Rosenfeld a] M. Rosenfeld, "In praise of the Gram matrix", preprint.

[Rosenamn 1992] J. Rosenman, "The rotation group and cancer treatment", Electronic message posted to sci.math (Internet newsgroup), Oct. 29, 1992.
[Seberry and Yamada 1992] J. Seberry and M. Yamada, "Hadamard matrices, sequences, and block designs", pp. 431-560 in Contemporary Design Theory (edited by J. H. Dinitz and D. R. Stinson), Wiley, New York, 1992.

[Seidel 1994] J. J. Seidel, "Discrete non-Euclidean geometry", Chapter 21 in Handbook of Incidence Geometry (edited by F. Buekenhout), North-Holland, Amsterdam, 1994.

[Stephanos 1879] C. Stephanos, "Sur les systèmes desmiques de trois tétraèdres", Bull. Sci. Math. 3 (1879), 424-456.

[Swayne et al. 1991] D. F. Swayne, D. Cook and A. Buja, "XGobi: Interactive dynamic graphics in the $\mathrm{X}$ window system with a link to S", pp. 1-8 in $A S A$ Proceedings Section on Statistical Graphics, Amer. Stat. Assoc., Alexandria, VA, 1991.

[Wong 1961] Y.-C. Wong, "Isoclinic $n$-Planes in Euclidean $2 n$-Space, Clifford Parallels in Elliptic $(2 n-1)-S p a c e$, and the Hurwitz Matrix Equations", Memoirs Amer. Math. Soc. 41, 1961.

[Wong 1967] Y.-C. Wong, "Differential geometry of Grassmann manifolds", Proc. Nat. Acad. Sci. USA 47 (1967), 589-594.

[Zanella 1995] C. Zanella, "Embeddings of Grassmann spaces", J. Geometry 52 (1995), 193-201.

John H. Conway, Department of Mathematics, Princeton University, Fine Hall, Washington Road, Princeton, NJ 08544 (conway@math.princeton.edu)

Ronald H. Hardin, Mathematical Sciences Research Center, AT\&T Research, 600 Mountain Avenue, Murray Hill, NJ 07974 (rhh@research.att.com)

Neil J. A. Sloane, Mathematical Sciences Research Center, AT\&T Research, 600 Mountain Avenue, Murray Hill, NJ 07974 (njas@research.att.com) — Corresponding author

Received December 12, 1995; accepted in revised form April 12, 1996 